\title{
Resolution of Operator Singularities via the Mixed-Variable Method
}

\author{
By
}

Sheng-Ming MA*

\begin{abstract}
This paper applies a modern method of singularity resolution in algebraic geometry to resolving singularities of integral operators in Fourier analysis. This is achieved by introducing a method of mixed variables that is equivalent to changing coordinates for integral operators. We decompose the integral operator into dyadic pieces via monomial transforms and the mixed-variable method so as to obtain its sharp estimates on different domains. These sharp estimates can be written in an elegant form in terms of continued fractions.
\end{abstract}

\section{§1. Introduction}

The monomial transform is a modern method for resolution of singularities in algebraic geometry. It emerged in the 1970s [1], [6] and is more efficient than the quadratic transform. Later on it was employed by Varchenko [1], [12] serving as the Jacobi transform for oscillatory integrals to resolve the singularities of their phase functions. In this way Varchenko established an intriguing link between the decay rate of an oscillatory integral and the Newton polyhedron of its phase function.

Nonetheless we do not have a routine similar to the Jacobi transform to change coordinates for integral operators, which constitutes a major difficulty in studying their singularities. In this paper we introduce a method of mixed

\footnotetext{
Communicated by M. Kashiwara. Received December 19, 2005. Revised January 19, 2007, March 29, 2007, June 16, 2008.

2000 Mathematics Subject Classification(s): 42B20, 47G10, 35S30, 35S05, 45P05, $47 \mathrm{~B} 38$.

The author is affiliated with the State Key Laboratory of Software Development Environment and supported by the Grant No. SKLSDE-07-004 under the 973 Grant No. 2005 CB321901.

*LMIB and Department of Mathematics, Beihang University, Beijing 100083, China.
} 
variables that is equivalent to changing coordinates for integral operators. In this way the machineries of algebraic geometry can be applied directly to studying the singularities of integral operators in Fourier analysis.

More specifically, we will study oscillatory integral operators $T_{\lambda}$ in the following form:

$$
T_{\lambda}(f)(x)=\int_{-\infty}^{\infty} e^{i \lambda P(x, y)} \varphi(x, y) f(y) d y,
$$

where $P(x, y)$ is a smooth phase function and $\varphi(x, y)$ is a smooth cutoff function supported in a neighborhood of the origin. We are interested in finding the decay rate of $T_{\lambda}$ defined as the best possible positive $\delta$ such that $\left\|T_{\lambda}\right\|_{2} \leq$ $C|\lambda|^{-\delta}$.

Hörmander [5] investigated the operator $T_{\lambda}$ in (1.1) with a non-degenerate phase function $P(x, y)$ and proved that its decay rate equals $d / 2$ for $x, y \in \mathbf{R}^{d}$. His method constitutes a cornerstone for the analysis of more general oscillatory integral operators.

For the operator $T_{\lambda}$ with a polynomial or real analytic phase function in the case of $x, y \in \mathbf{R}^{1}$, a notable progress was made in the thesis of Ma [7] and then appeared in the paper of Phong and Stein [11]. It asserts that the decay rate of $T_{\lambda}$ is determined by the Newton polygon of its phase, same as Varchenko's conclusion on oscillatory integrals. Nonetheless an obvious drawback of the method in [11] is the employment of Puiseux series for curve singularities, which cannot be generalized to algebraic varieties of higher dimensions. A note on this limitation is at the end of the "Introduction" of [11]. The Puiseux series also leads to a redundant discussion of its complex roots in [11]. Further, in order to have a summation of the infinite pieces of a dyadic decomposition, it is required that the estimates for the derivatives of the phase Hessian $P_{x y}^{\prime \prime}(x, y)$ be uniform on all pieces of the decomposition. It is excruciating to address this uniformity in terms of Puiseux series in [11].

In this paper we carry out dyadic decompositions via monomial transforms as per [7] so as to overcome these drawbacks. Our proof is based on two successive phases of decomposing the operator $T_{\lambda}$ :

$$
T_{\lambda}=\sum_{\alpha} T_{\alpha}=\sum_{\alpha} \sum_{k, j} T_{k j}^{\alpha} .
$$

After the first phase of the operator decompositions in (1.2), each $T_{\alpha}$ is an operator of mixed variables:

$$
T_{\alpha}(f)(x)=\int_{-\infty}^{\infty} e^{i \lambda P(x, y)} \varphi(x, y) \Phi\left(\left|Y_{\alpha}\right|\right) \Psi\left(\left|X_{\alpha}\right|\right) f(y) d y,
$$


where the variables $X_{\alpha}$ and $Y_{\alpha}$ are linked to the variables $x$ and $y$ by a finite composition of monomial transforms that resolves the singularities of the phase Hessian $P_{x y}^{\prime \prime}(x, y)$. The mixture of variables in the $T_{\alpha}$ is a new method that amounts to changing coordinates for integral operators.

The second phase of the operator decompositions in (1.2) is a routine dyadic partition of unity on the support of each operator $T_{\alpha}$. A summation balance between two kinds of estimates for the operator pieces $T_{k j}^{\alpha}$ in (1.2) leads to a sharp estimate for each $T_{\alpha}$.

In summary, we have the following stronger conclusion than those in [7] and [11]:

Theorem. Suppose the oscillatory integral operator $T_{\lambda}$ in (1.1) with $x, y \in \mathbf{R}^{1}$ has a real analytic phase function $P(x, y)$ and a smooth cutoff function $\varphi(x, y)$ supported in a sufficiently small neighborhood of the origin.

1. $T_{\lambda}$ has the following sharp estimate:

$$
\left\|T_{\lambda}\right\|_{2} \leq C \lambda^{-1 /[2(\Delta+1)]} .
$$

Here $(\Delta+1, \Delta+1)$ is exactly the intersection point of the bisector $y=x$ and the boundary of the Newton polygon of $P(x, y)$ excluding all its monadic monomials.

2. $T_{\lambda}$ can be decomposed into finite parts $T_{\lambda}=\sum_{\alpha} T_{\alpha}$ as in (1.2). For each operator $T_{\alpha}$ we have the following sharp estimate:

$$
\left\|T_{\alpha}\right\|_{2} \leq C_{\alpha} \lambda^{-1 /\left[2\left(\Delta_{\alpha}+1\right)\right]}
$$

where $\exists t(\alpha) \in \mathbf{N}$ such that $\Delta_{\alpha}=\frac{\left[b_{1}, b_{2}, \ldots, b_{t}\right]}{2\left[k_{1}, k_{2}, \ldots, k_{t}\right]}$ or $\frac{\left[b_{1}, b_{2}, \ldots, b_{t}^{\prime}\right]}{2\left[k_{1}, k_{2}, \ldots, k_{t}^{\prime}\right]}$ is the ratio of two finite continued fractions. These continued fractions are determined by a sequence of Newton polygons resolving the singularity of the phase Hessian $P_{x y}^{\prime \prime}(x, y)$. Here for $1<j \leq t, b_{j}, k_{j}, b_{j}^{\prime}$ and $k_{j}^{\prime}$ denote the intercept and the negative reciprocal of the slope respectively of a side of the $j$-th Newton polygon in the Newton polygon sequence (Please refer to (4.4) for more details). When $j=1$, the $\left(\frac{b_{1}}{2 k_{1}}, \frac{b_{1}}{2 k_{1}}\right)$ is exactly the intersection point of the bisector $y=x$ and the extended line of a side of the Newton polygon of $P_{x y}^{\prime \prime}(x, y) . C_{\alpha}$ is independent of $\lambda$. The $\Delta$ in (1.3) equals $\Delta=$ $\max _{\alpha}\left\{\Delta_{\alpha}\right\}$.

Here by "sufficiently small" in the assumption of the theorem, we mean that the size of the support of $\varphi(x, y)$ is determined by the Newton polygon of the phase Hessian $P_{x y}^{\prime \prime}(x, y)$, which will be clear from the proof. 
For simplicity we shall just denote $\|\cdot\|_{2}$ as $\|\cdot\|$ in the following context. As usual, we denote the sets of real numbers, rational numbers, integers and natural numbers as $\mathbf{R}, \mathbf{Q}, \mathbf{Z}$ and $\mathbf{N}$ respectively. In particular, we adopt the convention that $0 \notin \mathbf{N}$.

For a generic function $g(x, y)$, the notations $\operatorname{supp} g$ and $(\operatorname{supp} g)^{\circ}$ shall be used to denote the closure and interior of the set $\left\{(x, y) \in \mathbf{R}^{2} \mid g(x, y) \neq 0\right\}$ respectively.

\section{§2. Mixed-Variable Estimates}

It is difficult to change coordinates of integral operators through the traditional Jacobi transform because of the intricate impacts incurred on the function spaces of the operator. The method of mixed variables introduced in this section can overcome this difficulty so that we can directly apply the method of singularity resolutions in algebraic geometry to integral operators in analysis.

Definition 2.1. Horizontal and vertical connectedness.

A bounded set $D$ on the $(x, y)$-plane is defined as horizontally connected if for $\forall y_{0} \in \mathbf{R}$, the one-dimensional set $\left\{(x, y) \in D^{c} \mid y=y_{0}\right\}$ has at most two connected components. Similarly we can define vertically connected sets.

Henceforth we write $(x, y)=\mathcal{T}(X, Y)$ in the form of $x=x(X, Y)$ and $y=y(X, Y)$.

Lemma 2.1. $\quad$ Let $R$ be a rectangle on the $(X, Y)$-plane with sides parallel to the $X$ and $Y$ axes respectively and let $U$ be a neighborhood of $R$. Suppose that $\mathcal{T} \in C^{1}(U)$ is a diffeomorphism from $U$ to the $(x, y)$-plane with $\mathcal{T}^{-1} \in C^{1}(\mathcal{T}(U))$. If $\partial y(X, Y) / \partial X$ and $\partial y(X, Y) / \partial Y$ do not change sign for $(X, Y) \in \partial R$, then $\mathcal{T}(R)$ is horizontally connected.

Similarly, $\mathcal{T}(R)$ is vertically connected if $\partial x(X, Y) / \partial X$ and $\partial x(X, Y) / \partial Y$ do not change sign for $(X, Y) \in \partial R$.

Proof. Let the rectangle $R \supset \partial R$ and its vertices be $A(a, b), B(a, b+\eta)$, $C(a+\epsilon, b+\eta)$ and $D(a+\epsilon, b)$ respectively. The condition on $\partial y(X, Y) / \partial X$ and $\partial y(X, Y) / \partial Y$ indicates that the function $y(X, Y)$ is monotonic on the sides $A B \cup B C$ of $R$. Hence the boundary curve $\mathcal{T}(A B \cup B C)$ is horizontally connected. The same is true for the boundary curve $\mathcal{T}(C D \cup D A)$.

Let $H$ be a maximal horizontal segment in $\mathcal{T}(R)$ with two end points $E_{1}, E_{2} \in \partial \mathcal{T}(R)$ such that $E_{1} \neq E_{2}$ and their $y$-coordinate equals $y_{0}$. Here being "maximal" means that $H \supset\left\{\left(x, y_{0}\right) \in \mathcal{T}(R)\right\}$. If $H \backslash \mathcal{T}(R) \neq \emptyset$, then 
there exists an interval $\left[E_{1}^{\prime}, E_{2}^{\prime}\right] \subset H$ with $E_{1}^{\prime} \neq E_{2}^{\prime}$ such that the open interval $\left(E_{1}^{\prime}, E_{2}^{\prime}\right) \bigcap \mathcal{T}(R)=\emptyset$ and the set $\left\{E_{1}^{\prime}, E_{2}^{\prime}\right\} \subset \partial \mathcal{T}(R)$. First of all, suppose $H \backslash \mathcal{T}(R)=\left(E_{1}^{\prime}, E_{2}^{\prime}\right)$. Then it is easy to see that the generic case $H \bigcap \partial \mathcal{T}(R)=\left\{E_{1}, E_{1}^{\prime}, E_{2}, E_{2}^{\prime}\right\}$ contradicts the horizontal connectedness of the boundary curves $\mathcal{T}(A B \cup B C)$ and $\mathcal{T}(C D \cup D A)$. In the special cases such as $E_{1}=E_{1}^{\prime}, E_{2}=E_{2}^{\prime}$, or $H \bigcap \partial \mathcal{T}(R)$ contains an interval, we can vertically translate $H$ either upward or downward by a small distance. In this way these special cases can be reduced to the above generic case contradicting the horizontal connectedness of the boundary curves. More generally when $H \backslash \mathcal{T}(R) \supset\left(E_{1}^{\prime}, E_{2}^{\prime}\right)$, the above discussion still applies because we can shrink $H$ appropriately so that $H \backslash \mathcal{T}(R)=\left(E_{1}^{\prime}, E_{2}^{\prime}\right)$.

By symmetry the conclusion for the vertical connectedness can be immediately deduced.

In what follows we will write the map $(X, Y)=\mathcal{T}^{-1}(x, y)$ in the form of $X=X(x, y)$ and $Y=Y(x, y)$. We have the following lemma.

Lemma 2.2. $\quad$ Suppose that the rectangle $R$ in Lemma 2.1 has widths $\epsilon>0$ and $\eta>0$ in the $X$-direction and $Y$-direction respectively.

For a diffeomorphism $\mathcal{T}$ as in Lemma 2.1 whose partial derivatives satisfy the condition for the vertical connectedness, if $\exists \delta>0$ such that the partial derivatives of $\mathcal{T}^{-1}$ satisfy at least one of the following conditions for $(x, y) \in$ $\mathcal{T}(R)$ :

$$
|\partial X(x, y) / \partial y| \geq \epsilon / \delta, \quad|\partial Y(x, y) / \partial y| \geq \eta / \delta
$$

then the length of each vertical segment of $\mathcal{T}(R)$ is bounded by $\delta$.

Similarly, if the diffeomorphism $\mathcal{T}$ satisfies the condition for horizontal connectedness in Lemma 2.1, and $\exists \delta>0$ such that the partial derivatives of $\mathcal{T}^{-1}$ satisfy at least one of the following conditions for $(x, y) \in \mathcal{T}(R)$ :

$$
|\partial X(x, y) / \partial x| \geq \epsilon / \delta, \quad|\partial Y(x, y) / \partial x| \geq \eta / \delta,
$$

then the length of each horizontal segment of $\mathcal{T}(R)$ is bounded by $\delta$.

Proof. Suppose $\left(x, y_{j}\right) \in \partial \mathcal{T}(R)$ for $j=1,2$ with $y_{1}<y_{2}$. Then we have:

$$
\epsilon \geq\left|X\left(x, y_{2}\right)-X\left(x, y_{1}\right)\right|=\int_{y_{1}}^{y_{2}}|\partial X(x, y) / \partial y| d y \geq \epsilon\left(y_{2}-y_{1}\right) / \delta .
$$

This implies that the vertical segment connecting $\left(x, y_{1}\right)$ and $\left(x, y_{2}\right)$ has length $y_{2}-y_{1} \leq \delta$. 
Lemma 2.3. Let $R$ be a rectangle of the same magnitude as in Lemma 2.2. Suppose that $\mathcal{T}$ is a diffeomorphism as in Lemma 2.1 satisfying the condition for the vertical connectedness and the estimates in (2.1). Further, we assume that both $\mathcal{T}$ and $\mathcal{T}^{-1}$ are $C^{2}$-diffeomorphisms defined in a neighborhood of $R$ and $\mathcal{T}(R)$ respectively.

Suppose that for $l=1,2$ and $(x, y) \in \mathcal{T}(R)$, the partial derivatives of $\mathcal{T}^{-1}$ have upper bounds:

$$
\left|\partial^{l} X(x, y) / \partial y^{l}\right| \leq \epsilon / \delta^{l}, \quad\left|\partial^{l} Y(x, y) / \partial y^{l}\right| \leq \eta / \delta^{l}
$$

Further, we assume that the operator $T_{\lambda}$ in (1.1) with $x, y \in \mathbf{R}^{1}$ satisfies the following conditions:

1. Define $\chi:=\varphi \circ \mathcal{T}$ and assume that $\operatorname{supp} \chi \subset R$. Suppose that $\exists B>0$ such that for $k, l \in \mathbf{N} \cup\{0\}$ with $0 \leq k+l \leq 2$, the partial derivatives of $\chi(X, Y)$ satisfy:

$$
\left|\partial^{k} \partial^{l} \chi(X, Y) / \partial X^{k} \partial Y^{l}\right| \leq B /\left(\epsilon^{k} \eta^{l}\right)
$$

where the constant $B$ is independent of $\lambda, \epsilon$ and $\eta$.

2. Define $\mathcal{P}(X, Y):=P_{x y}^{\prime \prime}[x(X, Y), y(X, Y)]$. Suppose that $\exists \nu>0$ such that for $(X, Y) \in R$ and $k, l \in \mathbf{N} \cup\{0\}$ with $0 \leq k+l \leq 2$ :

$$
\nu \leq|\mathcal{P}(X, Y)|, \quad\left|\partial^{k} \partial^{l} \mathcal{P}(X, Y) / \partial X^{k} \partial Y^{l}\right| \leq \nu /\left(\epsilon^{k} \eta^{l}\right),
$$

where $\nu$ is independent of $\lambda$ but dependent on $\epsilon$ and $\eta$.

Then $\exists C>0$ such that the $T_{\lambda}$ can be extended to a bounded operator on $L^{2}\left(\mathbf{R}^{1}\right)$ :

$$
\left\|T_{\lambda}\right\| \leq C(\lambda \nu)^{-\frac{1}{2}}
$$

where the constant $C$ is independent of $\lambda, \nu, \epsilon$ and $\eta$.

Proof. To simplify notations, in what follows we use the same $C$ to denote all the constants that are independent of $\lambda, \nu, \epsilon$ and $\eta$.

Consider the kernel $K(x, y)$ of the integral operator $T_{\lambda} T_{\lambda}^{*}$ given by:

$$
K(x, y)=\int_{-\infty}^{\infty} e^{i \lambda[P(x, z)-P(y, z)]} \varphi(x, z) \bar{\varphi}(y, z) d z .
$$

A double integration by parts leads to the form:

$$
K(x, y)=\int_{-\infty}^{\infty} e^{i \lambda[P(x, z)-P(y, z)]} \mathcal{D}^{2}[\varphi(x, z) \bar{\varphi}(y, z)] d z
$$


with the operator $\mathcal{D}$ defined as $\mathcal{D} f=-(i \lambda)^{-1} \partial\left[\left(P_{z}^{\prime}(x, z)-P_{z}^{\prime}(y, z)\right)^{-1} f\right] / \partial z$.

(2.2) and (2.4) together with the chain rule for differentiation such as

$$
\begin{aligned}
\left|\partial P_{x y}^{\prime \prime}(x, y) / \partial y\right| \leq & |\partial \mathcal{P}(X, Y) / \partial X||\partial X(x, y) / \partial y|+ \\
& +|\partial \mathcal{P}(X, Y) / \partial Y||\partial Y(x, y) / \partial y|
\end{aligned}
$$

imply that:

$$
\left|\partial^{l} P_{x y}^{\prime \prime}(x, y) / \partial y^{l}\right| \leq C \nu / \delta^{l}, \quad l=0,1,2 .
$$

For $\theta_{1}, \theta_{2} \in[x, y]$ that satisfies the following equality:

$$
\partial\left[P_{z}^{\prime}(x, z)-P_{z}^{\prime}(y, z)\right]^{-1} / \partial z=(y-x)^{-1}\left[P_{\omega z}^{\prime \prime}\left(\theta_{1}, z\right)\right]^{-2} \partial P_{\omega z}^{\prime \prime}\left(\theta_{2}, z\right) / \partial z
$$

Lemma 2.1 ensures that $\left(\theta_{1}, z\right),\left(\theta_{2}, z\right) \in \mathcal{T}(R)$. Hence (2.4) and (2.6) indicate that:

$$
\left|\partial^{l}\left[P_{z}^{\prime}(x, z)-P_{z}^{\prime}(y, z)\right]^{-1} / \partial z^{l}\right| \leq C\left(\nu \delta^{l}|x-y|\right)^{-1}, \quad l=0,1,2,
$$

where the differentiation in the case of $l=2$ is performed in a similar way to the case of $l=1$ in (2.7).

Further, $(2.2),(2.3)$ and the chain rule for differentiation similar to $(2.5)$ imply:

$$
\left|\partial^{l} \varphi(x, y) / \partial y^{l}\right| \leq C / \delta^{l}, \quad l=0,1,2 .
$$

(2.9) together with (2.8) yield:

$$
\left|\mathcal{D}^{2}[\varphi(x, t) \bar{\varphi}(y, t)]\right| \leq C(\lambda \delta \nu|x-y|)^{-2},
$$

and hence the estimate:

$$
|K(x, y)| \leq C \delta^{-1}(\lambda \nu|x-y|)^{-2} .
$$

In addition, the conclusion of Lemma 2.2 indicates that $|K(x, y)| \leq C \delta$.

Balancing the above two estimates we have a new estimate:

$$
\int_{-\infty}^{\infty}|K(x, y)| d y \leq C \min _{\sigma>0}\left\{\delta \sigma+\int_{|y-x| \geq \sigma} \delta^{-1}(\lambda \nu|x-y|)^{-2} d y\right\}=C(\lambda \nu)^{-1},
$$

which implies that $\left\|T_{\lambda} T_{\lambda}^{*}\right\| \leq C(\lambda \nu)^{-1}$. 


\section{$\S 3 . \quad$ An Algorithm of Operator Decompositions}

In this section we integrate space partitions and operator decompositions with the algorithm of singularity resolutions in algebraic geometry. In this way we can introduce a partition of unity in a neighborhood of the origin to approximate each branch of a real analytic curve as well as to decompose the operator via the method of mixed variables.

\section{§3.1. An algorithm of singularity resolutions}

The elaboration of the resolution algorithm for real analytic functions in this section is almost verbatim to that of [9] for polynomials. The reason for the similarity is that the resolution algorithm for a real analytic function is only pertinent to the boundary of its Newton polygon. The repetition of the resolution algorithm in this section aims at the integrity of the proof as well as the convenience of the readers.

We define a positive quadrant with vertex $(a, b)$ as $\left\{(x, y) \in \mathbf{R}^{2} \mid x \geq\right.$ $a, y \geq b\}$. Given a real analytic function, consider the union of the positive quadrants whose vertices correspond to the exponents of its monomials.

Definition 3.1. Newton polygon.

The Newton polygon of a real analytic function is defined as the convex hull of the above union of positive quadrants.

The Newton polygon of a monomial $c x^{a} y^{b}(c \neq 0)$ is simply the positive quadrant with vertex $(a, b)$; whereas the Newton polygon of the polynomial $x^{3} y+x y^{3}-2 y^{4}$ is $\left\{(x, y) \in \mathbf{R}^{2} \mid x \geq 0, y \geq 1, x+y \geq 4\right\}$, which is the same as the Newton polygon of the real analytic function $x^{3} y+x y^{3}-2 y^{4}+$ $\sum_{\alpha \geq 0, \beta \geq 1, \alpha+\beta>4} c_{\alpha \beta} x^{\alpha} y^{\beta}$.

We denote a compact or noncompact face of a Newton polygon satisfying the equation $m x+n y=p$ as $[m x+n y=p]$ with $(m, n)=1$ if $m n \neq 0$. From the definition of the Newton polygon it is easy to see that $m, n, p \in \mathbf{N} \cup\{0\}$. We have the following lemma.

Lemma 3.1. Suppose $\{(a, b)\}=[m x+n y=p] \bigcap[\widetilde{m} x+\widetilde{n} y=\widetilde{p}]$ is $a$ vertex of the Newton polygon. If

$$
\operatorname{det}\left(\begin{array}{cc}
m & \widetilde{m} \\
n & \tilde{n}
\end{array}\right)>1,
$$


then there are a finite sequence of straight lines $r_{j} x+s_{j} y=q_{j}$ with $r_{j}, s_{j}, q_{j} \in$ $\mathbf{N}, 1<j<J$ passing through the vertex $(a, b)$ such that for $1 \leq k<J$,

$$
\operatorname{det}\left(\begin{array}{c}
r_{k} r_{k+1} \\
s_{k} s_{k+1}
\end{array}\right)=1 \quad \text { with }\left(\begin{array}{c}
r_{1} \\
s_{1}
\end{array}\right)=\left(\begin{array}{c}
m \\
n
\end{array}\right) \text { and }\left(\begin{array}{c}
r_{J} \\
s_{J}
\end{array}\right)=\left(\begin{array}{c}
\tilde{m} \\
\tilde{n}
\end{array}\right) \text {. }
$$

Proof. Evidently the matrix $\left(\begin{array}{cc}m & \widetilde{m} \\ n & \widetilde{n}\end{array}\right)$ has no inverse matrix in the integral domain Z. Hence we have $\left(\begin{array}{l}1 \\ 0\end{array}\right)\left(\right.$ or $\left.\left(\begin{array}{l}0 \\ 1\end{array}\right)\right)=\mu_{1}\left(\begin{array}{c}m \\ n\end{array}\right)+\mu_{2}\left(\begin{array}{c}\widetilde{m} \\ \widetilde{n}\end{array}\right)$ with $\mu_{1}, \mu_{2} \in \mathbf{Q} \backslash \mathbf{Z}$. Thus $\exists \lambda_{1}, \lambda_{2} \in \mathbf{Q} \cap(0,1)$ and a vector $\left(\begin{array}{l}r \\ s\end{array}\right)=\lambda_{1}\left(\begin{array}{c}m \\ n\end{array}\right)+\lambda_{2}\left(\begin{array}{c}\widetilde{m} \\ \widetilde{n}\end{array}\right)$ with $r, s \in \mathbf{N}$ such that

$$
\begin{aligned}
& \operatorname{det}\left(\begin{array}{cc}
m & r \\
n & s
\end{array}\right)=\lambda_{2} \operatorname{det}\left(\begin{array}{cc}
m & \tilde{m} \\
n & \widetilde{n}
\end{array}\right)<\operatorname{det}\left(\begin{array}{cc}
m & \widetilde{m} \\
n & \widetilde{n}
\end{array}\right), \\
& \operatorname{det}\left(\begin{array}{cc}
r & \widetilde{m} \\
s & \widetilde{n}
\end{array}\right)=\lambda_{1} \operatorname{det}\left(\begin{array}{cc}
m & \widetilde{m} \\
n & \widetilde{n}
\end{array}\right)<\operatorname{det}\left(\begin{array}{cc}
m & \widetilde{m} \\
n & \widetilde{n}
\end{array}\right) .
\end{aligned}
$$

The conclusion of the lemma follows from a decreasing induction on the integer values of the determinants.

We name the above finite sequence of straight lines $r_{j} x+s_{j} y=q_{j}$ with $r_{j}, s_{j}, q_{j} \in \mathbf{N}, 1<j<J$ satisfying (3.1) as a sequence of auxiliary lines at the vertex $(a, b)$.

Definition 3.2. Perfect Newton polygon.

A Newton polygon with auxiliary lines added to each of its vertices is named as a perfect Newton polygon.

Lemma 3.1 indicates that we can always refine a Newton polygon into a perfect Newton polygon. If we enumerate all the faces and auxiliary lines of the perfect Newton Polygon in increasing order of their slopes and denote them as $L_{\kappa}=\left[m_{\kappa} x+n_{\kappa} y=p_{\kappa}\right]$ respectively $(1 \leq \kappa \leq \rho)$, then each adjacent pair $L_{\kappa}$ and $L_{\kappa+1}$ satisfy

$$
\operatorname{det}\left(\begin{array}{cc}
m_{\kappa} & m_{\kappa+1} \\
n_{\kappa} & n_{\kappa+1}
\end{array}\right)=1
$$

for $1 \leq \kappa<\rho$. The proof of Lemma 3.1 shows that we always have $m_{\kappa}, n_{\kappa}, p_{\kappa} \in$ $\mathbf{N} \cup\{0\}$ with $1 \leq \kappa \leq \rho$.

A simple example is the Newton polygon of the real analytic function $P(x, y)=x y^{5}+x^{2} y^{2}+x^{5} y+\sum_{\alpha, \beta \in \mathbf{N}, 3 \alpha+\beta>8, \alpha+3 \beta>8} c_{\alpha \beta} x^{\alpha} y^{\beta}$ that consists of 3 vertices $\{(1,5),(2,2),(5,1)\}$ and 4 faces $\{[x=1],[3 x+y=8],[x+3 y=$ $8],[y=1]\}$. At the vertex $(1,5)=[x=1] \cap[3 x+y=8]$, the two faces 
satisfy $\operatorname{det}\left(\begin{array}{ll}1 & 3 \\ 0 & 1\end{array}\right)=1$ and thus it is unnecessary to add auxiliary lines to the vertex. The same is true for the vertex $(5,1)=[x+3 y=8] \cap[y=1]$. Nonetheless at the vertex $(2,2)=[3 x+y=8] \cap[x+3 y=8]$, the two faces satisfy $\operatorname{det}\left(\begin{array}{ll}3 & 1 \\ 1 & 3\end{array}\right)=8>1$ and hence we choose a sequence of integer vectors $\left(\begin{array}{l}1 \\ 1\end{array}\right)=\frac{1}{4}\left(\begin{array}{l}3 \\ 1\end{array}\right)+\frac{1}{4}\left(\begin{array}{l}1 \\ 3\end{array}\right),\left(\begin{array}{l}2 \\ 1\end{array}\right)=\frac{1}{2}\left(\begin{array}{l}3 \\ 1\end{array}\right)+\frac{1}{2}\left(\begin{array}{l}1 \\ 1\end{array}\right)$ and $\left(\begin{array}{l}1 \\ 2\end{array}\right)=\frac{1}{2}\left(\begin{array}{l}1 \\ 1\end{array}\right)+\frac{1}{2}\left(\begin{array}{l}1 \\ 3\end{array}\right)$ that satisfy $\operatorname{det}\left(\begin{array}{ll}3 & 2 \\ 1 & 1\end{array}\right)=\operatorname{det}\left(\begin{array}{ll}2 & 1 \\ 1 & 1\end{array}\right)=\operatorname{det}\left(\begin{array}{ll}1 & 1 \\ 1 & 2\end{array}\right)=\operatorname{det}\left(\begin{array}{ll}1 & 1 \\ 2 & 3\end{array}\right)=1$. These integer vectors $\left(\begin{array}{l}1 \\ 1\end{array}\right),\left(\begin{array}{l}2 \\ 1\end{array}\right)$ and $\left(\begin{array}{l}1 \\ 2\end{array}\right)$ correspond to auxiliary lines $[x+y=4],[2 x+y=6]$ and $[x+2 y=6]$ at the vertex $(2,2)$ respectively.

Based on each adjacent pair $L_{\kappa}$ and $L_{\kappa+1}$, which will be denoted as $\left\langle L_{\kappa}, L_{\kappa+1}\right\rangle$ with $1 \leq \kappa<\rho$, we have a monomial transform $(x, y)=\mathcal{T}_{\kappa}\left(X_{\kappa}, Y_{\kappa}\right)$ as follows:

$$
\mathcal{T}_{\kappa}:\left\{\begin{array}{l}
x=X_{\kappa}^{m_{\kappa}} Y_{\kappa}^{m_{\kappa+1}} \\
y=X_{\kappa}^{n_{\kappa}} Y_{\kappa}^{n_{\kappa+1}}
\end{array} ; \quad \mathcal{T}_{\kappa}^{-1}:\left\{\begin{array}{c}
X_{\kappa}=x^{n_{\kappa+1}} / y^{m_{\kappa+1}} \\
Y_{\kappa}=y^{m_{\kappa}} / x^{n_{\kappa}}
\end{array}\right.\right.
$$

whose exponents satisfy the condition (3.2). The $\mathcal{T}_{\kappa}$ is a bijective map and has an inverse $\mathcal{T}_{\kappa}^{-1}$ if we exclude all the axes.

From (3.3), we have the following relationship for $1 \leq \kappa<\rho-1$ :

$$
Y_{\kappa+1}=\frac{1}{X_{\kappa}} .
$$

Suppose the operator $T_{\lambda}$ in (1.1) with $x, y \in \mathbf{R}^{1}$ has a real analytic phase function $P(x, y)$. For the perfect Newton Polygon of the phase Hessian $P_{x y}^{\prime \prime}(x, y)$ defined as above, let $\left\langle L_{\kappa}, L_{\kappa+1}\right\rangle$ be an adjacent pair such that $L_{\kappa} \bigcap L_{\kappa+1}=$ $\left\{\left(a_{\kappa}, b_{\kappa}\right)\right\}$ with $a_{\kappa}, b_{\kappa} \in \mathbf{N} \cup\{0\}$. Then each monomial transform $\mathcal{T}_{\kappa}$ factorizes $P_{x y}^{\prime \prime}(x, y)$ as:

$$
\begin{aligned}
P_{x y}^{\prime \prime}(x, y) & =c_{\kappa} x^{a_{\kappa}} y^{b_{\kappa}}+\sum_{(\alpha, \beta) \in L_{\kappa} \cup L_{\kappa+1} \backslash\left\{\left(a_{\kappa}, b_{\kappa}\right)\right\}} c_{\alpha \beta} x^{\alpha} y^{\beta} \\
& +\sum_{(\delta, \gamma) \notin L_{\kappa} \cup L_{\kappa+1}} c_{\delta \gamma} x^{\delta} y^{\gamma}=X_{\kappa}^{p_{\kappa}} Y_{\kappa}^{p_{\kappa+1}} P_{1}\left(X_{\kappa}, Y_{\kappa}\right)
\end{aligned}
$$

with the exponents $\alpha, \beta, \delta, \gamma \in \mathbf{N} \cup\{0\}$. The coefficients $c_{\kappa}, c_{\alpha \beta}, c_{\delta \gamma} \in \mathbf{R} \backslash\{0\}$.

The $P_{1}(X, Y)$ in (3.5) is named as a partial transform and is a series of the following form:

$$
\begin{aligned}
c+ & \sum_{(\alpha, \beta) \in L_{\kappa} \backslash\left\{\left(a_{\kappa}, b_{\kappa}\right)\right\}} c_{\alpha \beta} Y^{l_{\alpha \beta}}+\sum_{(\alpha, \beta) \in L_{\kappa+1} \backslash\left\{\left(a_{\kappa}, b_{\kappa}\right)\right\}} c_{\alpha \beta} X^{\widetilde{l}_{\alpha \beta}} \\
& +\sum_{(\delta, \gamma) \notin L_{\kappa} \cup L_{\kappa+1}} c_{\delta \gamma} X^{\widetilde{l}_{\delta \gamma}} Y^{l_{\delta \gamma}}
\end{aligned}
$$


with the exponents $l_{\alpha \beta}, \widetilde{l}_{\alpha \beta}, l_{\delta \gamma}, \widetilde{l}_{\delta \gamma} \in \mathbf{N}$. The constant term $c$ is the coefficient $c_{\kappa}$ in $(3.5)$.

The monadic polynomial in $Y$ in (3.6) can be factorized as

$$
c+\sum_{(\alpha, \beta) \in L_{\kappa} \backslash\left\{\left(a_{\kappa}, b_{\kappa}\right)\right\}} c_{\alpha \beta} Y^{l_{\alpha \beta}}=Q(Y) \prod_{i}\left(Y-r_{i}\right)^{h_{i}}
$$

with $r_{i} \in \mathbf{R} \backslash\{0\}$. The monadic polynomial $Q$ stands for a product of quadratic polynomials that are not factorizable in the field $\mathbf{R}$

The monadic polynomial in $X$ in (3.6) also has a factorization

$$
c+\sum_{(\alpha, \beta) \in L_{\kappa+1} \backslash\left\{\left(a_{\kappa}, b_{\kappa}\right)\right\}} c_{\alpha \beta} X^{\widetilde{l}_{\alpha \beta}}=\widetilde{Q}(X) \prod_{j}\left(X-s_{j}\right)^{h_{j}}
$$

with $s_{j} \in \mathbf{R} \backslash\{0\}$. The polynomial $\widetilde{Q}$ is similar to the polynomial $Q$ in (3.7).

We make such kind of factorizations for each adjacent pair $\left\langle L_{\kappa}, L_{\kappa+1}\right\rangle$ with $1 \leq \kappa<\rho$. The $\left(0, r_{i}\right)$ or $\left(s_{j}, 0\right)$ obtained is named as a branch point except those $\left(0, r_{i}\right)$ of $\left\langle L_{1}, L_{2}\right\rangle$ and $\left(s_{j}, 0\right)$ of $\left\langle L_{\rho-1}, L_{\rho}\right\rangle$. Furthermore, because of the reciprocal relationship (3.4), for $1 \leq \kappa<\rho$ and $r \in \mathbf{R} \backslash\{0\}, Y_{\kappa+1}=r$ and $X_{\kappa}=1 / r$ represent the same branch of the initial analytic curve defined by the phase Hessian $\left\{P_{x y}^{\prime \prime}(x, y)=0\right\}$. Hence to avoid repetitions, hereafter we prescribe that $0<\left|r_{i}\right| \leq 1$ and $0<\left|s_{j}\right|<1$ for all the branch points of the form $\left(0, r_{i}\right)$ or $\left(s_{j}, 0\right)$.

For $1 \leq \kappa<\rho$, we denote:

$$
\Delta_{\kappa}:=\max \left\{\frac{p_{\kappa}}{m_{\kappa}+n_{\kappa}}, \frac{p_{\kappa+1}}{m_{\kappa+1}+n_{\kappa+1}}\right\}, \quad \Delta:=\max _{1 \leq \kappa<\rho}\left\{\Delta_{\kappa}\right\} .
$$

Then the bisector $y=x$ and the boundary of the original Newton Polygon intersect at $(\Delta, \Delta)$.

The algorithm of resolution of singularity continues as follows. Through the factorization in (3.7), we treat the partial transform $P_{1}(X, Y)$ in (3.6) as a new series:

$$
\widetilde{P}_{1}\left(X, Y-r_{i}\right):=P_{1}\left(X,\left(Y-r_{i}\right)+r_{i}\right)
$$

so as to address its singularity at the branch point $\left(0, r_{i}\right)$. We name the new series $\widetilde{P}_{1}\left(X, Y-r_{i}\right)$ as the reduced transform of the partial transform $P_{1}(X, Y)$. Then we construct the perfect Newton Polygon of the reduced transform $\widetilde{P}_{1}\left(X, Y-r_{i}\right)$ and deduce monomial transforms that are similar to those in (3.3). We enumerate these monomial transforms by a new subscript variable. For simplicity, we still use $\kappa$ to denote this new subscript variable.

The above resolution may lead to more branch points. With the above procedure repeated at each newly generated branch point, the branch points 
of the resolution procedure form a tree whose root is the origin of the $(x, y)$ plane. We use the same subscript variable $\kappa$ as the one in (3.2) to enumerate the different tree branches that are from the same branch point. The difference between this tree and a regular one is that each value of each subscript variable $\kappa$ of this tree further branches out to new branch points on the next level of the tree.

Following the above procedure we choose a branch on each level of the tree to obtain a path from its root to one of the last branch points. Hereafter we will use a subscript $t$ to enumerate the levels of the branch points on a path.

Without loss of generality, suppose that every branch point on a path takes the form $\left(0, r_{j}\right)$ with $1 \leq j \leq t$. Consider the following sequence of monomial transforms $\mathcal{T}_{1}, \cdots, \mathcal{T}_{t}$ based on the adjacent pairs $\left\langle L_{j}, L_{j}^{\prime}\right\rangle$ with $L_{j}=$ $\left[m_{j} x+n_{j} y=p_{j}\right]$ and $L_{j}^{\prime}=\left[m_{j}^{\prime} x+n_{j}^{\prime} y=p_{j}^{\prime}\right]$ such that $\operatorname{det}\left(\begin{array}{cc}m_{j} & m_{j}^{\prime} \\ n_{j} & n_{j}^{\prime}\end{array}\right)=1$ for $1 \leq j \leq t$ and $t \in \mathbf{N}$.

$$
\mathcal{T}_{1}:\left\{\begin{array}{l}
x=X_{1}^{m_{1}} Y_{1}^{m_{1}^{\prime}} \\
y=X_{1}^{n_{1}} Y_{1}^{n_{1}^{\prime}},
\end{array}, \cdots, \quad \mathcal{T}_{t}:\left\{\begin{array}{r}
X_{t-1}=X_{t}^{m_{t}} Y_{t}^{m_{t}^{\prime}} \\
Y_{t-1}-r_{t-1}=X_{t}^{n_{t}} Y_{t}^{n_{t}^{\prime}}
\end{array} .\right.\right.
$$

The composition of the above monomial transforms $\mathcal{T}_{1} \circ \cdots \circ \mathcal{T}_{t}$ factorizes the Hessian of the phase function $P_{x y}^{\prime \prime}(x, y)$ into a product:

$$
P_{x y}^{\prime \prime}(x, y)=\prod_{j=1}^{t} X_{j}^{p_{j}} Y_{j}^{p_{j}^{\prime}} P_{t}\left(X_{t}, Y_{t}\right):=\mathcal{P}\left(X_{t}, Y_{t}\right)
$$

with $P_{t}$ bearing a similar form to $P_{1}$ in (3.6).

It is easy to see that we can define the singularity height of a branch point in the same way as in Section 4 of [9] for polynomials. We can also prove verbatim like Lemma 4.3 of [9] that the singularity height of a reduced transform shall strictly decrease after a resolution step unless the reduced transform is either degenerate or nonsingular. Same as (4.1) of [9], the paradigm of a degenerate transform in $(X, Y-r)$ is:

$$
\left(Y-r-r_{1} X^{n}\right)^{h}+\sum_{\delta+n \gamma>n h} c_{\delta \gamma} X^{\delta}(Y-r)^{\gamma}
$$

whose Newton polygon has a single compact face of perfect power with its exponent $h$ being exactly the singularity height. Here $n, h \in \mathbf{N}$ and $\delta, \gamma \in$ $\mathbf{N} \cup\{0\}$. The coefficients $r, r_{1}, c_{\delta, \gamma} \in \mathbf{R} \backslash\{0\}$.

Hence same as in [9], it suffices to consider the degenerate transforms like (3.12) and after a monomial transform $X=X_{1}$ and $Y-r=X_{1}^{n} Y_{1}$, its partial 
transform takes the form:

$$
\left(Y_{1}-r_{1}\right)^{h}+\sum_{\delta+n \gamma>n h} c_{\delta \gamma} X_{1}^{\delta+n \gamma-n h} Y_{1}^{\gamma},
$$

which is the formula (5.1) in [9].

Nonetheless it is unnecessary to define the singularity index for real analytic functions as we did in Section 5 of [9] for polynomials. Instead, we can invoke the Weierstrass Preparation Theorem directly on (3.13) to obtain:

$$
\left[\left(Y_{1}-r_{1}\right)^{h}+\sum_{j=1}^{h} R_{j}\left(X_{1}\right)\left(Y_{1}-r_{1}\right)^{h-j}\right] E\left(X_{1}, Y_{1}-r_{1}\right),
$$

where $E\left(X_{1}, Y_{1}-r_{1}\right)$ is a real analytic function in the variables $\left(X_{1}, Y_{1}-r_{1}\right)$ with $E(0,0) \neq 0$. The $R_{j}\left(X_{1}\right)$ is a power series in $X_{1}$ such that $R_{j}(0)=0$ for $1 \leq j \leq h$. The reason for the definition of the singularity index in Section 5 of [9] is that the theme of [9] is algebraic instead of analytic whereas $E\left(X_{1}, Y_{1}-r_{1}\right)$ and $R_{j}\left(X_{1}\right)$ are series instead of polynomials.

When $h>1$, we expand each factor $\left(Y_{1}-r_{1}\right)^{j}$ in (3.14) as $\left[\left(Y_{1}-r_{1}+\right.\right.$ $\left.\left.R_{1}\left(X_{1}\right) / h\right)-R_{1}\left(X_{1}\right) / h\right]^{j}$ for $1 \leq j \leq h$. Then the factor inside the brackets in (3.14) becomes:

$$
\left(Y_{1}-r_{1}+R_{1}\left(X_{1}\right) / h\right)^{h}+\sum_{j=2}^{h} \widetilde{R}_{j}\left(X_{1}\right)\left(Y_{1}-r_{1}+R_{1}\left(X_{1}\right) / h\right)^{h-j}
$$

with $\widetilde{R}_{j}(0)=0$ for $2 \leq j \leq h$.

Now if $\widetilde{R}_{j}\left(X_{1}\right) \equiv 0$ for $2 \leq j \leq h$, then (3.15), and thus the factor inside the brackets in (3.14), are perfect powers and they represent a branch

$$
\left(Y_{1}-r_{1}+R_{1}\left(X_{1}\right) / h\right)^{h}
$$

of the initial analytic curve with multiplicity $h$.

However if $\exists j$ with $2 \leq j \leq h$ such that $\widetilde{R}_{j}\left(X_{1}\right) \neq 0$, then we make a coordinate change $X_{2}=X_{1}$ and $Y_{2}=Y_{1}-r_{1}+R_{1}\left(X_{1}\right) / h$ and (3.15) becomes an analytic function in the variables $\left(X_{2}, Y_{2}\right)$ :

$$
Y_{2}^{h}+\sum_{j=2}^{h} \widetilde{R}_{j}\left(X_{2}\right) Y_{2}^{h-j} .
$$

This analytic function cannot be a degenerate transform. As a result, its singularity height shall strictly decrease after another resolution step. 
Thus a recursive and finite repetition of the above algorithm shall lead to either a branch of the initial analytic curve like (3.16) whose multiplicity is strictly bigger than one, or a branch point whose singularity height equals one. The later case is equivalent to the degenerate transform (3.13) having singularity height $h=1$. Then after invoking the Weierstrass Preparation Theorem as above, (3.14) takes the following form instead:

$$
\left[Y_{1}-r_{1}+R_{1}\left(X_{1}\right)\right] E\left(X_{1}, Y_{1}-r_{1}\right)
$$

The above argument demonstrates that for each path in the resolution tree, $\exists t \in \mathbf{N}$ such that

$$
P_{t}\left(X_{t}, Y_{t}\right)=\left[Y_{t}-r\left(X_{t}\right)\right]^{h_{t}} E\left(X_{t}, Y_{t}-r_{t}\right)
$$

with $E\left(X_{t}, Y_{t}-r_{t}\right)$ being nonsingular as $E(0,0) \in \mathbf{R} \backslash\{0\} . r\left(X_{t}\right)$ is either a convergent power series with $r(0)=r_{t} \in \mathbf{R} \backslash\{0\}$, or a constant $r_{t} \in \mathbf{R} \backslash\{0\}$. Here $h_{t} \in \mathbf{N}$. In the case of (3.17) we define the branch point $\left(0, r_{t}\right)$ as a terminal branch point.

It is easy to see that at a terminal branch point $\left(s_{t}, 0\right), P_{t}$ has another possible form

$$
P_{t}\left(X_{t}, Y_{t}\right)=\left[X_{t}-s\left(Y_{t}\right)\right]^{h_{t}} \widetilde{E}\left(X_{t}-s_{t}, Y_{t}\right)
$$

Summarizing (3.11) and (3.17), $P_{x y}^{\prime \prime}(x, y)$ takes the following form at a terminal branch point $\left(0, r_{t}\right)$ :

$$
P_{x y}^{\prime \prime}(x, y)=\tilde{Y}_{t}^{h_{t}} \prod_{j=1}^{t} X_{j}^{p_{j}} Y_{j}^{p_{j}^{\prime}} E\left(X_{t}, Y_{t}-r_{t}\right):=\mathcal{P}\left(X_{t}, Y_{t}\right)
$$

where $h_{t} \in \mathbf{N}$. The variable $\tilde{Y}_{t}:=Y_{t}-r\left(X_{t}\right)$ as in (3.17) with $r(0)=r_{t} \in$ $\mathbf{R} \backslash\{0\}$.

By symmetry, at a terminal branch point $\left(s_{t}, 0\right), P_{x y}^{\prime \prime}(x, y)$ takes the form:

$$
P_{x y}^{\prime \prime}(x, y)=\widetilde{X}_{t}^{h_{t}} \prod_{j=1}^{t} X_{j}^{p_{j}} Y_{j}^{p_{j}^{\prime}} \widetilde{E}\left(X_{t}-s_{t}, Y_{t}\right):=\mathcal{P}\left(X_{t}, Y_{t}\right)
$$

with $\widetilde{X}_{t}:=X_{t}-s\left(Y_{t}\right)$ as in (3.18) such that $s(0)=s_{t} \in \mathbf{R} \backslash\{0\}$.

In the case of branch points $\left(s_{j}, 0\right)(1 \leq j<t)$, we simply replace $\left(X_{j}, Y_{j}-\right.$ $\left.r_{j}\right)$ by $\left(X_{j}-s_{j}, Y_{j}\right)$ in the $(j+1)$-th monomial transform of $(3.10)$. 


\section{§3.2. Partition of unity}

Suppose $\phi \in C^{\infty}((0,+\infty))$ is nonnegative and decreasing such that $\phi(x)=$ 1 for $0<x \leq 1$ and $\phi(x)=0$ for $x \geq 2$. We define $\phi_{k}(x):=\phi\left(2^{k} x\right)-\phi\left(2^{k+1} x\right)$ to have a dyadic partition of unity on $(0,+\infty)$ as $\sum_{k \in \mathbf{Z}} \phi_{k}(x)=1$.

To take advantage of (3.4), we define the conjugate function $\psi_{k}(x)$ of $\phi_{k}(x)$ as follows.

$$
\psi(x):=\phi(1 / x), \quad \psi_{k}(x):=\psi\left(2^{k} x\right)-\psi\left(2^{k-1} x\right)=\phi_{-k}(1 / x) .
$$

In this way we can rewrite the above dyadic partition of unity as:

$$
\sum_{k \in \mathbf{Z}} \phi_{k}(x)=\sum_{k \geq 0} \phi_{k}(x)+\sum_{j \geq 1} \psi_{j}(1 / x)=1 .
$$

For the simplicity of notations, we define

$$
\Phi_{n}(x):=\sum_{k \geq n} \phi_{k}(x) ; \quad \Psi_{n}(x):=\sum_{j \geq n} \psi_{j}(x) .
$$

From the above definitions, we can see that the supports of the functions $\Phi_{n}$ and $\Psi_{n}$ are $\operatorname{supp}\left(\Phi_{n}\right)=\operatorname{supp}\left(\Psi_{n}\right)=\left[0,2^{-n+1}\right]$. And we have $\Phi_{n}(x)=$ $\Psi_{n}(x)=1$ for $x \in\left(0,2^{-n}\right]$.

Lemma 3.2. $\quad$ For the monomial transforms $\mathcal{T}_{\kappa}$ with $1 \leq \kappa<\rho$ in (3.3), we define $c:=\max _{1 \leq \kappa \leq \rho}\left\{m_{\kappa}\right\}$ and $c^{\prime}:=\max _{1 \leq \kappa \leq \rho}\left\{n_{\kappa}\right\}$. Then we have a partition of unity on the rectangles $\left\{(x, y) \mid(|x|,|y|) \in\left(0,2^{-c}\right) \times\left(0,2^{-c^{\prime}}\right)\right\}$ :

$$
\sum_{\kappa=1}^{\rho-1} \Phi_{0}\left(\left|Y_{\kappa}\right|\right) \Psi_{1}\left(\left|X_{\kappa}\right|\right)=1
$$

Proof. For each monomial transform $\mathcal{T}_{\kappa}$ as in (3.3), which is based on an adjacent pair $\left\langle L_{\kappa}, L_{\kappa+1}\right\rangle$ with $1 \leq \kappa<\rho$, the determinant condition (3.2) indicates the following properties of the matrix per se in (3.2). First, the four entries of the matrix cannot be odd numbers simultaneously. Secondly, the entries on the same row or column of the matrix cannot be even numbers simultaneously. As a result, the monomial transform $\mathcal{T}_{\kappa}$ in (3.3) maps the four quadrants of the $\left(X_{\kappa}, Y_{\kappa}\right)$-plane to four sectors in the four quadrants of the $(x, y)$-plane respectively. Thus it suffices to prove the lemma in the first quadrant of the $(x, y)$-plane excluding the axes.

We begin by showing that:

$$
(0,1] \times(0,2] \subset \bigcup_{\kappa=1}^{\rho-1} \mathcal{T}_{\kappa}((0,1] \times(0,2]) .
$$


In fact, for $1 \leq \kappa<\rho$, the $\rho-1$ coordinate pairs $\left(X_{\kappa}, Y_{\kappa}\right)=\mathcal{T}_{\kappa}^{-1}(x, y)$ for $\forall(x, y) \in(0,1] \times(0,2]$ satisfy: (i) for $1<\kappa<\rho, X_{\kappa-1}=1 / Y_{\kappa}$; (ii) $X_{\rho-1}=x$ and $Y_{1}=y$. This implies that there would be a contradiction if for each $\kappa$ satisfying $1 \leq \kappa<\rho$, either $X_{\kappa}>1$ or $Y_{\kappa}>2$.

If we define $\mathcal{I}_{\kappa, \kappa^{\prime}}:=\mathcal{T}_{\kappa}((0,1] \times(0,2]) \cap \mathcal{T}_{\kappa^{\prime}}((0,1] \times(0,2])$, then for the $\mathcal{I}_{\kappa, \kappa^{\prime}}$ satisfying $\left|\kappa-\kappa^{\prime}\right|>1$,

$$
\mathcal{I}_{\kappa, \kappa^{\prime}} \bigcap\left(\left(0,2^{-c}\right) \times\left(0,2^{-c^{\prime}}\right)\right)=\emptyset .
$$

In fact, if $\exists \kappa$ with $1 \leq \kappa<\rho$ such that both $X_{\kappa} \geq \frac{1}{2}$ and $Y_{\kappa} \geq 1$ are true, then $x=X_{\kappa}^{m_{\kappa}} Y_{\kappa}^{m_{\kappa+1}} \geq 2^{-m_{\kappa}}, y=X_{\kappa}^{n_{\kappa}} Y_{\kappa}^{n_{\kappa+1}} \geq 2^{-n_{\kappa}}$ and hence $(x, y) \notin\left(0,2^{-c}\right) \times\left(0,2^{-c^{\prime}}\right)$. Now for $\kappa \leq p \leq \kappa^{\prime}$ and $\forall(x, y) \in \mathcal{I}_{\kappa, \kappa^{\prime}} \bigcap\left(\left(0,2^{-c}\right) \times\right.$ $\left.\left(0,2^{-c^{\prime}}\right)\right)$, consider the $\kappa^{\prime}-\kappa+1$ coordinate pairs $\left(X_{p}, Y_{p}\right)=\mathcal{T}_{p}^{-1}(x, y) . X_{\kappa} \leq 1$ and $Y_{\kappa+1}=1 / X_{\kappa} \geq 1$ indicate $X_{\kappa+1}<\frac{1}{2}$. We can proceed inductively on $p-\kappa$ to prove that $X_{\kappa^{\prime}-1}<\frac{1}{2}$. This contradicts $X_{\kappa^{\prime}-1}=1 / Y_{\kappa^{\prime}} \geq \frac{1}{2}$ since $(x, y) \in \mathcal{T}_{\kappa^{\prime}}((0,1] \times(0,2])$ indicates $Y_{\kappa^{\prime}} \leq 2$.

We are left with showing that (3.22) is an equality for $\forall(x, y) \in\left(0,2^{-c}\right) \times$ $\left(0,2^{-c^{\prime}}\right)$. In the above argument for (3.23), it is apparent that if we take $\kappa^{\prime}=\kappa+1$, we have

$$
\left[\mathcal{I}_{\kappa, \kappa+1} \bigcap\left(\left(0,2^{-c}\right) \times\left(0,2^{-c^{\prime}}\right)\right)\right] \subset\left[\mathcal{I}_{\kappa}\left(\left[\frac{1}{2}, 1\right] \times(0,1)\right) \bigcap \mathcal{T}_{\kappa+1}\left(\left(0, \frac{1}{2}\right) \times[1,2]\right)\right]
$$

for $1 \leq \kappa<\rho-1$. Then together with (3.23), (3.22) is reduced to an equality

$$
\Phi_{0}\left(Y_{\kappa}\right) \Psi_{1}\left(X_{\kappa}\right)+\Phi_{0}\left(Y_{\kappa+1}\right) \Psi_{1}\left(X_{\kappa+1}\right)=\Psi_{1}\left(X_{\kappa}\right)+\Phi_{0}\left(Y_{\kappa+1}\right)=1,
$$

which is exactly (3.21).

Corollary 3.1. For $d>1$, we have the following partition of unity on rectangles $\left\{(x, y) \mid(|x|,|y|) \in\left(0,2^{-2 c d}\right) \times\left(0,2^{-2 c^{\prime} d}\right)\right\}$ :

$$
\sum_{\kappa=1}^{\rho-1} \Phi_{0}\left(\left|Y_{\kappa}\right|\right) \Psi_{1}\left(\left|X_{\kappa}\right|\right)\left[1-\chi\left(X_{\kappa}, Y_{\kappa}\right)\right]=1,
$$

with $\chi\left(X_{\kappa}, Y_{\kappa}\right):=\left[1-\Phi_{d}\left(\left|Y_{\kappa}\right|\right)\right]\left[1-\Psi_{d}\left(\left|X_{\kappa}\right|\right)\right]$ supported on $\left( \pm 2^{-d}, \pm \infty\right) \times$ $\left( \pm 2^{-d}, \pm \infty\right)$ as a function in $X_{\kappa}$ and $Y_{\kappa}$.

The parameter $d$ in Corollary 3.1 is named as an adjustable parameter. The pair of parameters $\left(c, c^{\prime}\right)$ in Lemma 3.2 is named as a pair of exponential parameters associated with the origin $(0,0)$ of the $(x, y)$-plane.

It is easy to see that for every branch point in the resolution tree, there associated with a pair of exponential parameters. Hereafter for every branch 
point $\left(0, r_{j}\right)$ with $1 \leq j \leq t$ on a path of length $t$ in the resolution tree, we denote the pair of exponential parameters associated with it as $\left(c_{j}, c_{j}^{\prime}\right), 1 \leq j \leq t$.

In the Theorem in the Introduction, the "sufficiently small" neighborhood of the origin $(0,0)$ of the $(x, y)$-plane that supports the cutoff function $\varphi(x, y)$ of the operator $T_{\lambda}$ is determined by the above adjustable and exponential parameters.

\section{§3.3. An algorithm of operator decompositions}

For the operator $T_{\lambda}$ in (1.1) with $x, y \in \mathbf{R}^{1}$ and a real analytic phase function $P(x, y)$, suppose that the support of its cutoff function $\varphi(x, y)$ satisfies $\operatorname{supp} \varphi \subset\left(-2^{-2 c d}, 2^{-2 c d}\right) \times\left(-2^{-2 c^{\prime} d}, 2^{-2 c^{\prime} d}\right)$. Here the $\left(c, c^{\prime}\right)$ is the exponential parameter pair defined as in Lemma 3.2; whereas the $d$ is the adjustable parameter defined as in Corollary 3.1.

We invoke Corollary 3.1 and the method of mixed variables to decompose the operator $T_{\lambda}$ as follows:

$$
T_{\lambda}=\sum_{\kappa=1}^{\rho-1} T_{\kappa}+T^{\circ}, \quad T_{\kappa}(f)(x):=\int_{0}^{\infty} e^{i \lambda P(x, y)} \widetilde{\varphi}\left(X_{\kappa}, Y_{\kappa}\right) f(y) d y
$$

where

$$
\widetilde{\varphi}\left(X_{\kappa}, Y_{\kappa}\right):=\Phi_{0}\left(\left|Y_{\kappa}\right|\right) \Psi_{1}\left(\left|X_{\kappa}\right|\right)\left[1-\chi\left(X_{\kappa}, Y_{\kappa}\right)\right] \varphi(x, y)
$$

and $P(x, y)$ is the real analytic phase function of the $T_{\lambda}$. The operator $T^{\circ}$ has support function $\chi_{\{x y=0\}}(x, y) \varphi(x, y)$ with $\chi_{\{x y=0\}}(x, y)$ being the characteristic function of the two axes. Since the two axes have measure zero, it is easy to see that $\left\|T^{\circ}\right\|=0$. Thus the operator $T^{\circ}$ has no contribution to $\left\|T_{\lambda}\right\|$. In the following operator decompositions we shall disregard such kind of operators with zero-measure support.

In order to isolate the branch point $\left(0, r_{i}\right)$, we define a cutoff function

$$
\varphi_{r_{i}}\left(X_{\kappa}, Y_{\kappa}\right):=\Phi_{2 c_{1}^{\prime} d}\left(\left|Y_{\kappa}-r_{i}\right|\right) \Psi_{2 c_{1} d}\left(\left|X_{\kappa}\right|\right)
$$

with $d$ being the same adjustable parameter as the one in Corollary 3.1 and (3.25) for the support function $\chi\left(X_{\kappa}, Y_{\kappa}\right)$. The pair of exponential parameters $\left(c_{1}, c_{1}^{\prime}\right)$ are defined almost the same as the exponential parameter pair $\left(c, c^{\prime}\right)$ in Lemma 3.2 except that they are associated with the branch point $\left(0, r_{i}\right)$. Evidently $\exists d \in \mathbf{N}$ such that $\operatorname{supp} \varphi_{r_{i}} \bigcap \operatorname{supp} \varphi_{r_{i^{\prime}}}=\emptyset$ for $i \neq i^{\prime}$. Similarly we define a cutoff function $\varphi_{s_{j}}$ to isolate the branch point $\left(s_{j}, 0\right)$. 
Now let $\varphi_{0}:=\widetilde{\varphi} \prod_{i}\left(1-\varphi_{r_{i}}\right) \prod_{j}\left(1-\varphi_{s_{j}}\right)$. We can further decompose each operator $T_{\kappa}$ in (3.24) into:

$$
T_{\kappa}=T_{0}+\sum_{i} T_{r_{i}}+\sum_{j} T_{s_{j}}
$$

with the cutoff functions of the operators $T_{0}, T_{r_{i}}$ and $T_{s_{j}}$ being $\varphi_{0}, \widetilde{\varphi} \varphi_{r_{i}}$ and $\widetilde{\varphi} \varphi_{s_{j}}$ respectively. In this way the operator $T_{0}$ accounts for the singularities of the origin $(0,0)$ of the $\left(X_{\kappa}, Y_{\kappa}\right)$-plane; whereas $T_{r_{i}}$ and $T_{s_{j}}$ account for the singularities of the branch points $\left(0, r_{i}\right)$ and $\left(s_{j}, 0\right)$ respectively.

The support of $\varphi_{0}$ might consist of several connected regions on which we have identical arguments and estimates for the operator $T_{0}$. Hence we just take the one holding the origin of the $\left(X_{\kappa}, Y_{\kappa}\right)$-plane as $\operatorname{supp} \varphi_{0}$. For the operators $T_{r_{i}}$ and $T_{s_{j}}$ in (3.27), we treat them like the operator $T_{\lambda}$ and, following the second step of the singularity resolution, repeat the decomposition in (3.24). Then we repeat the operator decomposition in (3.27) for another time at the branch points on the next level of the resolution tree.

In this way we have an algorithm of operator decompositions that decomposes the operators $T_{r_{i}}$ and $T_{s_{j}}$ in (3.27) into a finite sum of operators. All these operators can be classified into two kinds of operators that will be denoted as $T_{0}^{t}$ and $T_{r}^{t}$ respectively.

The first kind of operators, which is denoted as $T_{0}^{t}$, accounts for the singularity at the origin $(0,0)$ of the $\left(X_{t}, Y_{t}\right)$-plane and is defined in the same way as the operator $T_{0}$ in (3.27). Its cutoff function $\varphi_{0}^{t}$ is defined as:

$$
\varphi_{0}^{t}:=\widetilde{\varphi}_{t} \prod_{i}\left(1-\varphi_{r_{i}^{t}}\right) \prod_{j}\left(1-\varphi_{s_{j}^{t}}\right)
$$

with

$$
\widetilde{\varphi}_{t}\left(X_{t}, Y_{t}\right):=\Phi_{0}\left(\left|Y_{t}\right|\right) \Psi_{1}\left(\left|X_{t}\right|\right)\left[1-\chi\left(X_{t}, Y_{t}\right)\right] \widetilde{\varphi}_{r_{t-1}}\left(X_{t-1}, Y_{t-1}\right)
$$

being similar to the cutoff function $\widetilde{\varphi}$ of the operator $T_{\kappa}$ in (3.25). The cutoff function $\widetilde{\varphi}_{r_{t-1}}$ in (3.29) is similar to the cutoff function $\varphi(x, y)$ in $(3.25)$ and is defined as

$$
\widetilde{\varphi}_{r_{t-1}}:=\Phi_{2 c_{t-1}^{\prime} d}\left(\left|Y_{t-1}-r_{t-1}\right|\right) \Psi_{2 c_{t-1} d}\left(\left|X_{t-1}\right|\right) \widetilde{\varphi}_{t-1}
$$

with $d$ being the same adjustable parameter as the one defined in Corollary 3.1, (3.25) and (3.26). The pair of exponential parameters $\left(c_{t-1}, c_{t-1}^{\prime}\right)$ are defined almost the same as the exponential parameter pair $\left(c_{1}, c_{1}^{\prime}\right)$ in $(3.26)$ except that they are associated with the branch point $\left(0, r_{t-1}\right)$. The cutoff function 
$\widetilde{\varphi}_{r_{t-1}}$ is also similar to the cutoff function of the operator $T_{r_{i}}$ in (3.27). Here $\left(X_{t-1}, Y_{t-1}-r_{t-1}\right)$ are the variables of the monomial transform $\mathcal{T}_{t}$ in (3.10). The cutoff function $\widetilde{\varphi}_{t-1}$ in (3.30) is recursively defined like the cutoff function $\widetilde{\varphi}_{t}$ in (3.29). The cutoff function $\varphi_{r_{i}^{t}}$ in (3.28) is simply

$$
\varphi_{r_{i}^{t}}:=\Phi_{2 c_{t}^{\prime} d}\left(\left|Y_{t}-r_{i}^{t}\right|\right) \Psi_{2 c_{t} d}\left(\left|X_{t}\right|\right)
$$

with the adjustable parameter $d$ being the same as the one in (3.30). The exponential parameter pair $\left(c_{t}, c_{t}^{\prime}\right)$ in (3.31) are associated with the branch point $\left(0, r_{t}\right)$. The cutoff function $\varphi_{s_{i}^{t}}$ in (3.28) is defined similarly to the cutoff function $\varphi_{r_{i}^{t}}$ in (3.31).

The other kind of operator, which is denoted as $T_{r}^{t}$, accounts for the singularity of a terminal branch point $\left(0, r_{i}^{t}\right)$ and has similar definition to the operator $T_{r_{i}}$ or $T_{s_{j}}$ in (3.27). Its cutoff function $\varphi_{r}^{t}$ is defined as:

$$
\varphi_{r}^{t}:=\widetilde{\varphi}_{t} \Phi_{d}\left(\left|\widetilde{Y}_{t}\right|\right) \Psi_{d}\left(\left|X_{t}\right|\right)
$$

with the adjustable parameter $d$ being the same as in (3.29) and (3.31). Here the cutoff function $\widetilde{\varphi}_{t}$ is defined as in (3.29) and $\widetilde{Y}_{t}$ is defined as in (3.19). Please note that when the branch point $\left(0, r_{i}^{t}\right)$ is a terminal branch point as in (3.32), the cutoff function (3.28) should take a slightly different form, i.e., the cutoff function $\varphi_{r_{i}^{t}}$ defined in (3.31) should be substituted by the cutoff function $\Phi_{d}\left(\left|\tilde{Y}_{t}\right|\right) \Psi_{d}\left(\left|X_{t}\right|\right)$ in (3.32). Similarly at a terminal branch point $\left(s_{j}^{t}, 0\right)$, the cutoff function of the operator $T_{r}^{t}$ takes the form:

$$
\varphi_{r}^{t}:=\widetilde{\varphi}_{t} \Phi_{d}\left(\left|Y_{t}\right|\right) \Psi_{d}\left(\left|\widetilde{X}_{t}\right|\right)
$$

with the adjustable parameter $d$ being the same as in (3.32).

\section{§4. Some Preliminary Estimates}

In this section we shall introduce some preliminary estimates as well as carry out the second phase of the operator decompositions in (1.2), which are routine dyadic decompositions.

\section{$\S 4.1$. Some preliminary estimates}

For $t>1$, we define a function $\Gamma_{t}:=\prod_{i=1}^{t-1}\left(Y_{j}-r_{j}\right)$ and substitute $X_{j}-s_{j}$ for $Y_{j}-r_{j}$ in the case of the branch point $\left(s_{j}, 0\right)$; we define $\Gamma_{1}:=1$. 
Lemma 4.1. Let $l=1,2$. For the cutoff function $\varphi_{0}^{t}$ defined as in (3.28), as well as its adjustable parameter $d, \exists d \in \mathbf{N}$ such that the following estimates hold on $\left(\operatorname{supp} \varphi_{0}^{t}\right)^{\circ}$ :

$$
\left|\partial^{l} X_{t} / \partial y^{l}\right| \leq C\left|X_{t} /\left(y \Gamma_{t}\right)^{l}\right|, \quad\left|\partial^{l} Y_{t} / \partial y^{l}\right| \leq C\left|Y_{t} /\left(y \Gamma_{t}\right)^{l}\right|
$$

For the cutoff function $\varphi_{r}^{t}$ defined in (3.32) or (3.33) as well as its adjustable parameter $d, \exists d \in \mathbf{N}$ such that the following estimates hold on $\left(\operatorname{supp} \varphi_{r}^{t}\right)^{\circ}$ :

$$
\left|\partial^{l} X_{t} / \partial y^{l}\right| \leq C\left|X_{t} /\left(y \Gamma_{t}\right)^{l}\right|, \quad\left|\partial^{l} \widetilde{Z}_{t} / \partial y^{l}\right| \leq C\left|\widetilde{Z}_{t} /\left(y \Gamma_{t}\right)^{l} \widetilde{Z}_{t}\right|
$$

with $\widetilde{Z}_{t}=\widetilde{Y}_{t}$ in the case of the terminal branch point $\left(0, r_{t}\right)$ as in (3.19) whereas $\widetilde{Z}_{t}=\widetilde{X}_{t}$ in the case of the terminal branch point $\left(s_{t}, 0\right)$ as in $(3.20)$. Here we keep the variable $\widetilde{Z}_{t}$ in the upper bound of the second estimate on purpose for later usages.

If we substitute $x$ for $y$ in the above estimates, the conclusions still hold.

Proof. The conclusion follows from an induction on $t$. When $t=2$, we apply the chain rule for differentiations to the composition of monomial transforms in (3.10) to obtain $\frac{\partial Y_{2}}{\partial y}=n_{2} m_{1}^{\prime} \frac{Y_{2}}{y}+m_{2} m_{1} \frac{Y_{2} Y_{1}}{\left(Y_{1}-r_{1}\right) y}$, whose estimate is straightforward.

Lemma 4.2. Let $(k, l) \in\{(1,0),(0,1)\}$. In the case of the branch point $\left(0, r_{t-1}\right)$, and for the cutoff functions $\varphi_{0}^{t}$ and $\varphi_{r}^{t}$ defined in (3.28) and (3.32) or (3.33) respectively with their adjustable parameter $d, \exists d \in \mathbf{N}$ such that the following estimates:

$$
\left|\partial^{k} \partial^{l} Y_{t} / \partial x^{k} \partial y^{l}\right| \geq C\left|Y_{t} /\left(x^{k} y^{l} \Gamma_{t}\right)\right|, \quad\left|\partial^{k} \partial^{l} \widetilde{Z}_{t} / \partial x^{k} \partial y^{l}\right| \geq C\left|\widetilde{Z}_{t} /\left(x^{k} y^{l} \widetilde{Z}_{t} \Gamma_{t}\right)\right|
$$

hold on $\left(\operatorname{supp} \varphi_{0}^{t}\right)^{\circ}$ and $\left(\operatorname{supp} \varphi_{r}^{t}\right)^{\circ}$ respectively. The variable $\widetilde{Z}_{t}:=\widetilde{Y}_{t}$ in the case of the terminal branch point $\left(0, r_{t}\right)$ as in (3.19) whereas $\widetilde{Z}_{t}:=\widetilde{X}_{t}$ in the case of the terminal branch point $\left(s_{t}, 0\right)$ as in (3.20). Here we keep the variable $\widetilde{Z}_{t}$ in the lower bound of the second estimate on purpose for later usages. In the case of the branch point $\left(s_{t-1}, 0\right)$, we simply substitute $X_{t}$ for $Y_{t}$ and the first estimate still holds on $\left(\operatorname{supp} \varphi_{0}^{t}\right)^{\circ}$; whereas the second estimate does not change on $\left(\operatorname{supp} \varphi_{r}^{t}\right)^{\circ}$.

When $t=1$ we have lower bounds $\left|\partial_{x} X_{1}\right| \geq C\left|X_{1} / x\right|$ and $\left|\partial_{y} Y_{1}\right| \geq C\left|Y_{1} / y\right|$.

Proof. Apart from the chain rule for differentiation and an induction on $t$, our proof depends on the exponents of the monomial transforms in (3.10) being nonzero. In fact, we always have $m_{j} \cdot n_{j}^{\prime} \neq 0$ for $\forall 1 \leq j \leq t$. And same 
as the definition of the branch point under (3.7), we rule out both $\left(0, r_{j}\right)$ of $\left\langle L_{1}, L_{2}\right\rangle$ and $\left(s_{j}, 0\right)$ of $\left\langle L_{\rho-1}, L_{\rho}\right\rangle$ for each Newton polygon in our resolution tree and accordingly, we have either $n_{j} \neq 0$ or $m_{j}^{\prime} \neq 0$, depending on the form of the branch point being $\left(0, r_{j}\right)$ or $\left(s_{j}, 0\right)$ with $1 \leq j \leq t$. To obtain the second estimate we can use the estimates in Lemma 4.1.

The proof of Lemma 4.3 as follows is similar to those of Lemma 4.1 and Lemma 4.2 .

Lemma 4.3. Let $1 \leq k+l \leq 2$ with $k, l \in \mathbf{N} \cup\{0\}$. In the case of the branch points $\left(0, r_{j}\right)$ with $1 \leq j<t$, and for the cutoff functions $\varphi_{0}^{t}$ and $\varphi_{r}^{t}$ defined in (3.28) and (3.32) or (3.33) respectively with their adjustable parameter $d, \exists d \in \mathbf{N}$ such that the following estimates:

$$
\begin{aligned}
& \left|\partial^{k} \partial^{l} X_{j} / \partial X_{t}^{k} \partial Y_{t}^{l}\right| \leq C\left|X_{j} /\left(X_{t}^{k} Y_{t}^{l}\right)\right|, \\
& \left|\partial^{k} \partial^{l} Y_{j} / \partial X_{t}^{k} \partial Y_{t}^{l}\right| \leq C\left|\left(Y_{j}-r_{j}\right) /\left(X_{t}^{k} Y_{t}^{l}\right)\right|
\end{aligned}
$$

hold on $\left(\operatorname{supp} \varphi_{0}^{t}\right)^{\circ}$; whereas in the cases of the terminal branch points $\left(0, r_{t}\right)$ or $\left(s_{t}, 0\right)$ on $\left(\operatorname{supp} \varphi_{r}^{t}\right)^{\circ}$, we substitute $\widetilde{Y}_{t}$ for $Y_{t}$ or substitute $\widetilde{X}_{t}$ for $X_{t}$ respectively and the above estimates still hold.

For the same $k, l$ as above and $z=x$ or $z=y, \exists d \in \mathbf{N}$ such that the following estimates:

$$
\left|\partial^{k} \partial^{l} z / \partial X_{t}^{k} \partial Y_{t}^{l}\right| \leq C\left|z /\left(X_{t}^{k} Y_{t}^{l}\right)\right|
$$

hold on $\left(\operatorname{supp} \varphi_{0}^{t}\right)^{\circ}$; whereas in the cases of the terminal branch points $\left(0, r_{t}\right)$ or $\left(s_{t}, 0\right)$ on $\left(\operatorname{supp} \varphi_{r}^{t}\right)^{\circ}$, we substitute $\widetilde{Y}_{t}$ for $Y_{t}$ or substitute $\widetilde{X}_{t}$ for $X_{t}$ respectively and the above estimates still hold.

Without loss of generality, suppose that each branch point takes the form $\left(0, r_{j}\right)$ with $1 \leq j \leq t$. Hence the exponents of the monomial transforms in (3.10) satisfy $n_{j} \neq 0$ for $1 \leq j \leq t$. And we always have $m_{j} \cdot n_{j}^{\prime} \neq 0$ for $1 \leq j \leq t$.

For $1 \leq j<t$, let $\alpha_{j}:=\prod_{i=j+1}^{t} m_{i}$ and $\alpha_{t}:=1$. For the cutoff functions $\varphi_{0}^{t}$ and $\varphi_{r}^{t}$ defined in (3.28) and (3.32) respectively with their adjustable parameter $d$, it is easily seen from (3.10), (3.11) and (3.19) that $\exists d \in \mathbf{N}$ such that

$$
\left|\mathcal{P}\left(X_{t}, Y_{t}\right)\right| \sim\left|X_{t}\right|^{\nu_{t}}\left|Y_{t}\right|^{\nu_{t}^{\prime}} \text { and }\left|\mathcal{P}\left(X_{t}, Y_{t}\right)\right| \sim\left|X_{t}\right|^{\nu_{t}}\left|\widetilde{Y}_{t}\right|^{h_{t}}
$$

hold on $\operatorname{supp} \varphi_{0}^{t}$ and $\operatorname{supp} \varphi_{r}^{t}$ respectively. Here $\nu_{t}:=\sum_{j=1}^{t} \alpha_{j} p_{j}$ and $\nu_{t}^{\prime}:=$ $\frac{m_{t}^{\prime}}{m_{t}}\left(\nu_{t}-p_{t}\right)+p_{t}^{\prime}$. The $\Pi_{1} \sim \Pi_{2}$ in (4.1) means that $\exists C>0$ such that $C \Pi_{2} \leq$ $\Pi_{1} \leq C \Pi_{2}$. 
Similar to (4.1), it is also clear that $\exists d \in \mathbf{N}$ such that

$$
|x y| \Gamma_{t}^{2} \sim X_{t}^{2 \mu_{t}} Y_{t}^{2 \mu_{t}^{\prime}} \quad \text { and }|x y| \widetilde{Y}_{t}^{2} \Gamma_{t}^{2} \sim X_{t}^{2 \mu_{t}} \widetilde{Y}_{t}^{2}
$$

hold on $\operatorname{supp} \varphi_{0}^{t}$ and $\operatorname{supp} \varphi_{r}^{t}$ respectively. Here $\mu_{t}:=\sum_{j=1}^{t} \alpha_{j} \widetilde{n}_{j}$ and $\mu_{t}^{\prime}:=$ $\frac{m_{t}^{\prime}}{m_{t}}\left(\mu_{t}-\widetilde{n}_{t}\right)+\widetilde{n}_{t}^{\prime}$ with $\widetilde{n}_{j}=n_{j}, \widetilde{n}_{j}^{\prime}=n_{j}^{\prime}$ for $j>1$ and $\widetilde{n}_{1}=\frac{m_{1}+n_{1}}{2}, \widetilde{n}_{1}^{\prime}=\frac{m_{1}^{\prime}+n_{1}^{\prime}}{2}$. In the second estimate of (4.2) we keep the $\tilde{Y}_{t}^{2}$ on purpose for later usages.

In particular, in the case of the terminal branch point $\left(s_{t}, 0\right)$, it is easily seen from (3.10) and (3.20) that $\exists d \in \mathbf{N}$ such that (4.1) and (4.2) becomes:

$$
\left|\mathcal{P}\left(X_{t}, Y_{t}\right)\right| \sim\left|\widetilde{X}_{t}\right|^{h_{t}}\left|Y_{t}\right|^{\nu_{t}^{\prime}} \text { and }|x y| \widetilde{X}_{t}^{2} \Gamma_{t}^{2} \sim \widetilde{X}_{t}^{2} Y_{t}^{2 \mu_{t}^{\prime}}
$$

on $\operatorname{supp} \varphi_{r}^{t}$ with the cutoff function $\varphi_{r}^{t}$ defined as in (3.33). Here in the second estimate we keep the $\widetilde{X}_{t}^{2}$ on purpose for later usages.

For the simplicity of notations, let us define:

$$
\Delta_{t}:=\nu_{t} /\left(2 \mu_{t}\right)=\frac{\left[b_{1}, b_{2}, \ldots, b_{t}\right]}{2\left[k_{1}, k_{2}, \ldots, k_{t}\right]}, \quad \Delta_{t}^{\prime}:=\nu_{t}^{\prime} /\left(2 \mu_{t}^{\prime}\right)=\frac{\left[b_{1}, b_{2}, \ldots, b_{t}^{\prime}\right]}{2\left[k_{1}, k_{2}, \ldots, k_{t}^{\prime}\right]}
$$

In particular when $t=1$, we have $\Delta_{1}^{\prime}:=b_{1}^{\prime} /\left(2 k_{1}^{\prime}\right)=p_{1}^{\prime} /\left(m_{1}^{\prime}+n_{1}^{\prime}\right)$. Here $b_{1}=p_{1}$, $b_{1}^{\prime}=p_{1}^{\prime}, k_{1}=\widetilde{n}_{1}$ and $k_{1}^{\prime}=\widetilde{n}_{1}^{\prime}$; and when $1<j \leq t, b_{j}=p_{j} / m_{j}$ and $k_{j}=\widetilde{n}_{j} / m_{j}$ are the abscissa intercept and the negative reciprocal of the slope of the side $L_{j}$ of the $j$-th Newton polygon in (3.10) respectively; whereas $b_{j}^{\prime}=p_{j}^{\prime} / m_{j}^{\prime}$ and $k_{j}^{\prime}=\widetilde{n}_{j}^{\prime} / m_{j}^{\prime}$ are the abscissa intercept and the negative reciprocal of the slope of the side $L_{j}^{\prime}$ respectively. The $\left[b_{1}, b_{2}, \ldots, b_{t}\right]$ and $\left[k_{1}, k_{2}, \ldots, k_{t}\right]$ denote the continued fractions:

$$
\begin{aligned}
& {\left[p_{1}, \frac{p_{2}}{m_{2}}, \ldots, \frac{p_{t}}{m_{t}}\right]:=p_{1}+\frac{p_{2}+\frac{p_{3}+\cdots+\frac{p_{t}}{m_{t}}}{m_{3}}}{m_{2}}} \\
& {\left[\widetilde{n}_{1}, \frac{n_{2}}{m_{2}}, \ldots, \frac{n_{t}}{m_{t}}\right]:=\widetilde{n}_{1}+\frac{n_{2}+\frac{n_{3}+\cdots+\frac{n_{t}}{m_{t}}}{m_{3}}}{m_{2}}}
\end{aligned}
$$

Please note that the premise for the above definition is that for $1 \leq j \leq t$, each branch point has the form $\left(0, r_{j}\right)$. Since $(4.4)$ is the exponents of the second conclusion of the Theorem as in (1.4), in what follows we shall elaborate on the other cases of the branch points in details. When the branch points have the form $\left(s_{j}, 0\right)$ for $1 \leq t_{1} \leq j \leq t_{2}<t-1$, we substitute the variables $\left(X_{j}, Y_{j}-r_{j}\right)$ in the $(j+1)$-th monomial transform of $(3.10)$ by the variables $\left(X_{j}-s_{j}, Y_{j}\right)$. We 
also substitute the $Y_{j}-r_{j}$ in $\Gamma_{t}=\prod_{j=1}^{t-1}\left(Y_{j}-r_{j}\right)$ by $X_{j}-s_{j}$. Accordingly, we revise the $\Delta_{t}$ and $\Delta_{t}^{\prime}$ defined in (4.4) as follows. The $b_{t_{1}}$ and $k_{t_{1}}$ are substituted by $b_{t_{1}}^{\prime}$ and $k_{t_{1}}^{\prime}$ respectively; If $t_{2}>t_{1}$, then for $t_{1}<j \leq t_{2}$, the $b_{j}$ and $k_{j}$ are substituted by the ordinate intercept $p_{j}^{\prime} / n_{j}^{\prime}$ and the negative slope $m_{j}^{\prime} / n_{j}^{\prime}$ of $L_{j}^{\prime}$ respectively; The $b_{t_{2}+1}$ and $k_{t_{2}+1}$ are substituted by the ordinate intercept $p_{t_{2}+1} / n_{t_{2}+1}$ and the negative slope $m_{t_{2}+1} / n_{t_{2}+1}$ of $L_{t_{2}+1}$ respectively.

When $t_{2}=t-1$, besides the above revisions, we have to further revise the $\Delta_{t}^{\prime}$ defined in (4.4), i.e., to substitute the $b_{t}^{\prime}$ and $k_{t}^{\prime}$ by the ordinate intercept $p_{t}^{\prime} / n_{t}^{\prime}$ and the negative slope $m_{t}^{\prime} / n_{t}^{\prime}$ of $L_{t}^{\prime}$ respectively. Whereas in the case of $t_{1}<t_{2}=t$, besides all the above revisions, we have to substitute $\Delta_{t}^{\prime}$ for $\Delta_{t}$ on $\operatorname{supp} \varphi_{r}^{t}$.

Finally, in the case of $t_{1}=t_{2}=t$, it suffices to substitute $\Delta_{t}^{\prime}$ for $\Delta_{t}$ on $\operatorname{supp} \varphi_{r}^{t}$.

The monomial transform $\mathcal{T}_{1}$ in (3.10) represents a monomial transform $\mathcal{T}_{\kappa}$ in (3.3) for some subscript variable $\kappa$. And we have the following lemma.

Lemma 4.4. Let $t>1$. For the cutoff functions $\varphi_{0}^{t}$ and $\varphi_{r}^{t}$ defined in (3.28) and (3.32) or (3.33) respectively, the following inequalities hold on $\operatorname{supp} \varphi_{0}^{t}$ as well as on $\operatorname{supp} \varphi_{r}^{t}$ in the case of the terminal branch point $\left(0, r_{t}\right)$ :

$$
\max \left\{h_{t} / 2, \Delta_{t}^{\prime}\right\} \leq \Delta_{t} \leq \Delta_{\kappa} \leq \Delta .
$$

In the case of the terminal branch point $\left(s_{t}, 0\right)$ on $\operatorname{supp} \varphi_{r}^{t}$, we have:

$$
h_{t} / 2 \leq \Delta_{t}^{\prime} \leq \Delta_{\kappa} \leq \Delta .
$$

The above conclusions are independent of the form of the branch points being $\left(0, r_{j}\right)$ or $\left(s_{j}, 0\right)$ for $1 \leq j \leq t-1$.

When $t=1$, we have $h_{1} / 2 \leq \max \left\{\Delta_{1}, \Delta_{1}^{\prime}\right\}=\Delta_{\kappa} \leq \Delta$.

Proof. Some calculations and the following observations can lead to the conclusion. In fact, the monadic polynomial in (3.7) implies that $h_{i}+1 \leq N_{\kappa}$ with $N_{\kappa}$ being the number of integral points on the face $L_{\kappa}$ of the Newton polygon. And $N_{\kappa} \leq \min \left\{\frac{p_{\kappa}}{n_{\kappa}}, \frac{p_{\kappa}}{m_{\kappa}}\right\}+1 \leq \frac{2 p_{\kappa}}{m_{\kappa}+n_{\kappa}}+1$. Using the subscript of the monomial transform $\mathcal{T}_{1}$ in $(3.10)$, this amounts to $h_{1}+1 \leq \frac{2 p_{1}}{m_{1}+n_{1}}+1=2 \Delta_{1}+1$. Similarly in the case of the branch point $\left(s_{1}, 0\right)$, we can obtain from (3.8) that $h_{1}+1 \leq \frac{2 p_{1}^{\prime}}{m_{1}^{\prime}+n_{1}^{\prime}}+1=2 \Delta_{1}^{\prime}+1$. Altogether we have $h_{1} / 2 \leq \max \left\{\Delta_{1}, \Delta_{1}^{\prime}\right\}=$ $\Delta_{\kappa} \leq \Delta$. Here $\Delta_{\kappa}$ and $\Delta$ are defined as in (3.9). When $t>1$, it suffices to prove the conclusion when each branch point, including the terminal branch point, takes the form $\left(0, r_{j}\right)$ for $1 \leq j \leq t$. For $\forall j$ with $1<j \leq t$, we can 
define similar quantities $h_{j}$ and $N_{j}$ and they also satisfy $h_{j}+1 \leq N_{j}$. In addition, since $\left(0, h_{j-1}\right)$ is the initial vertex of the perfect Newton Polygon at the branch point $\left(0, r_{j-1}\right)$, we have $N_{j} \leq p_{j} / \widetilde{n}_{j}+1 \leq h_{j-1}+1$. Summarizing the above inequalities in a recursive way, we can obtain $h_{t} \leq p_{j} / \widetilde{n}_{j} \leq 2 \Delta_{\kappa}$ for $\forall j$ with $1 \leq j \leq t$. Then notice that in the definition of $\Delta_{t}$ in (4.4), we have $b_{j}=k_{j}\left(p_{j} / \widetilde{n}_{j}\right)$ for $1 \leq j \leq t$ and thus the inequality $\frac{h_{t}}{2} \leq \Delta_{t} \leq \Delta_{\kappa}$. The inequality $\Delta_{t}^{\prime} \leq \Delta_{t}$ can be proved in a similar way. In particular, we need to substitute $p_{t}=m_{t} \widetilde{c}_{t}+n_{t} \widetilde{d}_{t}$ and $p_{t}^{\prime}=m_{t}^{\prime} \widetilde{c}_{t}+n_{t}^{\prime} \widetilde{d}_{t}$ into the definitions of $\nu_{t}, \mu_{t}, \nu_{t}^{\prime}$ and $\mu_{t}^{\prime}$ respectively. Here the $\left(\widetilde{c}_{t}, \widetilde{d}_{t}\right)$ is the vertex of the adjacent pair $\left\langle L_{t}, L_{t}^{\prime}\right\rangle$, i.e., $\left\{\left(\widetilde{c}_{t}, \widetilde{d}_{t}\right)\right\}:=L_{t} \cap L_{t}^{\prime}$. It is evident that $\widetilde{d}_{t} \leq h_{t-1} \leq p_{j} / \widetilde{n}_{j}$ for $1 \leq j \leq t-1$.

Corollary 4.1. Let $t>1$. For the cutoff functions $\varphi_{0}^{t}$ and $\varphi_{r}^{t}$ defined in (3.28) and (3.32) or (3.33) respectively with their adjustable parameter $d$, $\exists d \in \mathbf{N}$ and $\exists C>0$ such that $\left|\mathcal{P}\left(X_{t}, Y_{t}\right)\right| \geq C\left(|x y| \Gamma_{t}^{2}\right)^{\Delta_{t}}$ holds on $\operatorname{supp} \varphi_{0}^{t}$; whereas $\left|\mathcal{P}\left(X_{t}, Y_{t}\right)\right| \geq C\left(|x y| \widetilde{Y}_{t}^{2} \Gamma_{t}^{2}\right)^{\Delta_{t}}$ holds in the case of the terminal branch point $\left(0, r_{t}\right)$, or $\left|\mathcal{P}\left(X_{t}, Y_{t}\right)\right| \geq C\left(|x y| \widetilde{X}_{t}^{2} \Gamma_{t}^{2}\right)^{\Delta_{t}^{\prime}}$ holds in the case of the terminal branch point $\left(s_{t}, 0\right)$ on $\operatorname{supp} \varphi_{r}^{t}$.

When $t=1$, the estimate $\left|\mathcal{P}\left(X_{1}, Y_{1}\right)\right| \geq C|x y|^{\Delta_{\kappa}}$ holds on $\operatorname{supp} \varphi_{0}^{1}$ with $\Delta_{\kappa}=\max \left\{\Delta_{1}, \Delta_{1}^{\prime}\right\}$ defined as in (3.9). In the case of the terminal branch point $\left(0, r_{1}\right)$ the estimate $\left|\mathcal{P}\left(X_{1}, Y_{1}\right)\right| \geq C\left(|x y| \widetilde{Y}_{1}^{2}\right)^{\Delta_{\kappa}}$, or in the case of the terminal branch point $\left(s_{1}, 0\right)$ the estimate $\left|\mathcal{P}\left(X_{1}, Y_{1}\right)\right| \geq C\left(|x y| \widetilde{X}_{1}^{2}\right)^{\Delta_{\kappa}}$ holds on $\operatorname{supp} \varphi_{r}^{1}$.

Proof. (4.1), (4.2), (4.3), (4.4) and the above lemma immediately yield the conclusion.

\section{$\S 4.2$. Dyadic decompositions}

We have the following dyadic operator decompositions:

$$
T_{0}^{t}=\sum_{k \geq 0, j \geq 1} S_{k j}^{t}, \quad T_{r}^{t}=\sum_{k \geq d, j \geq d} \widetilde{S}_{k j}^{t}
$$

that correspond to the cutoff function decompositions $\varphi_{0}^{t}=\sum_{k \geq 0, j \geq 1} \varphi_{k j}^{t}$ and $\varphi_{r}^{t}=\sum_{k \geq d, j \geq d} \widetilde{\varphi}_{k j}^{t}$ respectively as follows.

We decompose the cutoff function $\varphi_{0}^{t}$ defined in (3.28) through a dyadic decomposition of its factor

$$
\Phi_{0}\left(\left|Y_{t}\right|\right) \Psi_{1}\left(\left|X_{t}\right|\right)=\sum_{k \geq 0, j \geq 1} \phi_{k}\left(\left|Y_{t}\right|\right) \psi_{j}\left(\left|X_{t}\right|\right) .
$$


The cutoff functions $\varphi_{r}^{t}$ in (3.32) and (3.33) are similarly decomposed via a dyadic decomposition of their factors

$$
\begin{aligned}
& \Phi_{d}\left(\left|\tilde{Y}_{t}\right|\right) \Psi_{d}\left(\left|X_{t}\right|\right)=\sum_{k \geq d, j \geq d} \phi_{k}\left(\left|\tilde{Y}_{t}\right|\right) \psi_{j}\left(\left|X_{t}\right|\right) ; \\
& \Phi_{d}\left(\left|Y_{t}\right|\right) \Psi_{d}\left(\left|\tilde{X}_{t}\right|\right)=\sum_{k \geq d, j \geq d} \phi_{k}\left(\left|Y_{t}\right|\right) \psi_{j}\left(\left|\tilde{X}_{t}\right|\right) .
\end{aligned}
$$

These are the second phase of the operator decompositions in (1.2).

The proof of Lemma 4.5 as follows is similar to those of Lemma 4.1 and Lemma 4.2 .

Lemma 4.5. For the $k, j, t$ and adjustable parameter $d$ in (4.5), $\exists d \in \mathbf{N}$ and $\exists c_{k j}^{t}>0$ dependent on $k, j$ and $t$ such that the estimates $\left|\partial x / \partial X_{t}\right| \geq c_{k j}^{t}$ and $\left|\partial y / \partial Y_{t}\right| \geq c_{k j}^{t}$ hold on $\operatorname{supp} \varphi_{k j}^{t}$.

Further, we have either $\left|\partial x / \partial Y_{t}\right| \geq c_{k j}^{t}$ or $\partial x / \partial Y_{t} \equiv 0$ and the same holds for $\partial y / \partial X_{t}$ on $\operatorname{supp} \varphi_{k j}^{t}$.

In the case of the terminal branch point $\left(0, r_{t}\right)$ or $\left(s_{t}, 0\right)$ on $\operatorname{supp} \widetilde{\varphi}_{k j}^{t}$, we substitute $\widetilde{Y}_{t}$ for $Y_{t}$ or substitute $\widetilde{X}_{t}$ for $X_{t}$ in the above estimates respectively.

The conclusion of the lemma is independent of the form of the branch points being $\left(0, r_{j}\right)$ or $\left(s_{j}, 0\right)$ for $1 \leq j \leq t-1$.

\section{$\S 5 . \quad$ Summation Balance and Sharp Estimates}

We invoke Lemma 2.3 to estimate the operators $S_{k j}^{t}$ and $\widetilde{S}_{k j}^{t}$ in (4.5). The final conclusion of the Theorem is a balance between the summations of these estimates. Then we make a scale transform in terms of the parameter $\lambda$ to prove the sharpness of our estimates.

\section{§5.1. Summation balance}

In what follows we first discuss the operator $S_{k j}^{t}$ in details since the estimate of the operator $\widetilde{S}_{k j}^{t}$ is almost verbatim. For the estimate of the operator $\widetilde{S}_{k j}^{t}$, we only mention its difference from that of the operator $S_{k j}^{t}$.

Since $\left|X_{t}\right| \sim 2^{-j}$ and $\left|Y_{t}\right| \sim 2^{-k}$ hold on $\operatorname{supp} \varphi_{k j}^{t}$, as a result of (4.1) and similar to (4.2), $\exists C_{1}, C_{2}>0$ independent of $k$ and $j$, and $\exists \nu, \delta_{1}, \delta_{2}>0$ dependent on $k$ and $j$, such that for $(\alpha, \beta) \in\{(1,0),(0,1)\}$, the following estimates hold on $\operatorname{supp} \varphi_{k j}^{t}$.

$$
C_{1} \nu \leq\left|\mathcal{P}\left(X_{t}, Y_{t}\right)\right| \leq C_{2} \nu, \quad C_{1} \delta_{1}^{\alpha} \delta_{2}^{\beta} \leq\left|x^{\alpha} y^{\beta} \Gamma_{t}\right| \leq C_{2} \delta_{1}^{\alpha} \delta_{2}^{\beta} .
$$


Lemma 4.2, (5.1) and Lemma 2.2 imply that the horizontal segment and vertical segment of the support of the kernel of the operator $S_{k j}^{t}$ on the $(x, y)$ plane, which is the image of $\operatorname{supp} \varphi_{k j}^{t}$ under $\mathcal{T}_{1} \circ \cdots \circ \mathcal{T}_{t}$ in $(3.10)$, can be controlled by $\delta_{1}$ and $\delta_{2}$ respectively. Hence it is straightforward that:

$$
\left\|S_{k j}^{t}\right\| \leq C\left(\delta_{1} \delta_{2}\right)^{1 / 2} .
$$

Next we verify that the operator $S_{k j}^{t}$ satisfies the conditions of Lemma 2.3 under the diffeomorphism $\mathcal{T}=\mathcal{T}_{1} \circ \cdots \circ \mathcal{T}_{t}$ in $(3.10)$.

Lemma 4.3 indicates that the cutoff function $\varphi_{k j}^{t}$ of the operator $S_{k j}^{t}$ satisfies the estimates in (2.3) with $\epsilon=2^{-j}$ and $\eta=2^{-k}$. Further, it follows from Lemma 4.5 that $\partial x\left(X_{t}, Y_{t}\right) / \partial X_{t}$ and $\partial x\left(X_{t}, Y_{t}\right) / \partial Y_{t}$ do not change sign for $\left(X_{t}, Y_{t}\right) \in \operatorname{supp} \varphi_{k j}^{t}$, and thus satisfy the conditions of Lemma 2.1 for the vertical connectedness. In addition, according to (3.11), Lemma 4.3 and (5.1) we can directly verify that $\mathcal{P}\left(X_{t}, Y_{t}\right)$ satisfies the estimates of (2.4). Finally, the estimates in Lemma 4.1 guarantee the condition (2.2). In summary, the operator $S_{k j}^{t}$ satisfies the conditions of Lemma 2.3 and we have:

$$
\left\|S_{k j}^{t}\right\| \leq C(\lambda \nu)^{-1 / 2} \leq C \lambda^{-1 / 2}\left(\delta_{1} \delta_{2}\right)^{-\Delta_{t} / 2},
$$

where the constant $C$ is independent of $\lambda, k, j, \nu, \delta_{1}$ and $\delta_{2}$. The second estimate is based on $C_{2} \nu \geq\left|\mathcal{P}\left(X_{t}, Y_{t}\right)\right| \geq C\left(|x y| \Gamma_{t}^{2}\right)^{\Delta_{t}} \geq C^{\prime}\left(\delta_{1} \delta_{2}\right)^{\Delta_{t}}$ according to Corollary 4.1 and (5.1).

Similar to (4.2), we can prove that for $(\alpha, \beta) \in\{(0,1),(1,0),(1,1)\}$ and $i=$ $1,2, \exists A_{i}, B_{i}>0$ depending only on the exponents of the monomial transforms in (3.10) such that $\left(\left|x \Gamma_{t}\right|\right)^{\alpha}\left(\left|y \Gamma_{t}\right|\right)^{\beta} \sim 2^{-\left(\alpha A_{1}+\beta A_{2}\right) k-\left(\alpha B_{1}+\beta B_{2}\right) j}$ on $\operatorname{supp} \varphi_{k j}^{t}$. Let $A:=A_{1}+A_{2}$ and $B:=B_{1}+B_{2}$. From (5.1) and (5.2), it is evident that $\left\|S_{k j}^{t}\right\| \leq C^{\prime} 2^{-(A k+B j) / 2}$. Moreover, it is easy to verify that $\operatorname{det}\left(\begin{array}{ll}A_{1} & A_{2} \\ B_{1} & B_{2}\end{array}\right)=$ $\left(m_{1}-n_{1}\right) D$ with $D:=\alpha_{1} / m_{t}>0$. The $\alpha_{1}$ is defined above (4.1) in the case of the branch points bearing the form $\left(0, r_{j}\right)$ for $1 \leq j<t$. The $m_{1}$ and $n_{1}$ are the exponents of the monomial transform $\mathcal{T}_{1}$ in (3.10). Thus when $m_{1} \neq n_{1}$, if both $\left|j-j^{\prime}\right| \geq h A$ and $A k+B j=A k^{\prime}+B j^{\prime}$ hold for $h>0$, then $\left|\left(A_{i} k^{\prime}+B_{i} j^{\prime}\right)-\left(A_{i} k+B_{i} j\right)\right| \geq h D(i=1,2)$. Hence $\exists h_{0}>0$ satisfying the above condition such that $\varphi_{k j}^{t}(x, z) \bar{\varphi}_{k^{\prime} j^{\prime}}^{t}(y, z)=\bar{\varphi}_{k j}^{t}(z, x) \varphi_{k^{\prime} j^{\prime}}^{t}(z, y)=0$. Now Cotlar's lemma implies that $\left\|\sum_{A k+B j=\ell} S_{k j}^{t}\right\| \leq C h_{0}\left\|S_{k j}^{t}\right\|$.

According to (4.5) we have $\left\|T_{0}^{t}\right\| \leq \sum_{\ell}\left\|\sum_{A k+B j=\ell} S_{k j}^{t}\right\| \leq C h_{0} \sum_{\ell} 2^{-\ell / 2}$.

When $m_{1}=n_{1}$, we have $A_{1}=A_{2}, B_{1}=B_{2}$ and thus $\delta_{1}=\delta_{2}$ and $x \sim y$. In this case the operator $\sum_{A k+B j=\ell} S_{k j}^{t}$ should be substituted by a single operator $S_{k j}^{t}$ satisfying $A k+B j=\ell$.

Thus the conclusion for the operator $T_{0}^{t}$ can be derived from a summation 
balance between (5.2) and (5.3).

$$
\left\|T_{0}^{t}\right\| \leq \min _{N>1}\left\{C_{1} \sum_{\ell \geq N} 2^{-\ell / 2}, C_{2} \lambda^{-\frac{1}{2}} \sum_{\ell<N} 2^{\left(\ell \Delta_{t}\right) / 2}\right\} \leq C \lambda^{-1 /\left[2\left(\Delta_{t}+1\right)\right]} .
$$

The estimates for the operator $\widetilde{S}_{k j}^{t}$ is almost verbatim except that the second estimate in (5.1) now becomes:

$$
C_{1} \delta_{1}^{\alpha} \delta_{2}^{\beta} \leq\left|x^{\alpha} y^{\beta} \widetilde{Z}_{t} \Gamma_{t}\right| \leq C_{2} \delta_{1}^{\alpha} \delta_{2}^{\beta},
$$

where $\widetilde{Z}_{t}=\widetilde{Y}_{t}$ or $\widetilde{X}_{t}$ depending on the terminal branch point being $\left(0, r_{t}\right)$ as in $(3.19)$ or $\left(s_{t}, 0\right)$ as in $(3.20)$.

Similarly we can obtain the estimate:

$$
\left\|T_{r}^{t}\right\| \leq C \lambda^{-1 /\left[2\left(\Delta_{t}+1\right)\right]} \quad \text { or } \quad\left\|T_{r}^{t}\right\| \leq C \lambda^{-1 /\left[2\left(\Delta_{t}^{\prime}+1\right)\right]}
$$

depending on the terminal branch point being $\left(0, r_{t}\right)$ as in $(3.19)$ or $\left(s_{t}, 0\right)$ as in (3.20). When $t=1$, we have estimates $\left\|T_{0}^{1}\right\| \leq C \lambda^{-1 /\left[2\left(\Delta_{\kappa}+1\right)\right]}$ and $\left\|T_{r}^{1}\right\| \leq C \lambda^{-1 /\left[2\left(\Delta_{\kappa}+1\right)\right]}$ with $\Delta_{\kappa}$ defined as in (3.9).

The (5.4) and (5.5) are the second conclusion of the Theorem as in (1.4).

\section{$\S 5.2$. Sharp estimates}

The sharpness of the estimates for the operators $T_{0}^{t}$ and $T_{r}^{t}$ as in (5.4) and (5.5) can be deduced from a scale transform in terms of the parameter $\lambda$ within the operators.

In fact, since we excluded the monadic monomials from the analytic phase function $P(x, y)$ of the operators $T_{0}^{t}$ and $T_{r}^{t}$, the two functions $P(x, y) /(x y)$ and $P_{x y}^{\prime \prime}(x, y)$ have identical Newton polygons. Hence the estimate $|P(x, y)| \leq$ $C|x y|\left|X_{1}\right|^{p_{1}}\left|Y_{1}\right|^{p_{1}^{\prime}}$ holds on both $\operatorname{supp} \varphi_{0}^{t}$ and $\operatorname{supp} \varphi_{r}^{t}$ for the operators $T_{0}^{t}$ and $T_{r}^{t}$. Here we used the same notations as in (3.11) and (3.19). As a result, there is an adjustable parameter $d \in \mathbf{N}$ such that, the following estimates:

$$
|P(x, y)| \leq C\left|X_{t}\right|^{\delta_{t}}\left|Y_{t}\right|^{\delta_{t}^{\prime}}, \quad \text { and } \quad|P(x, y)| \leq C\left|X_{t}\right|^{\delta_{t}} \text { or } C\left|Y_{t}\right|^{\delta_{t}^{\prime}}
$$

hold on $\operatorname{supp} \varphi_{0}^{t}$, and on $\operatorname{supp} \varphi_{r}^{t}$ in the case of the terminal branch point $\left(0, r_{t}\right)$ or $\left(s_{t}, 0\right)$ as in $(3.19)$ or $(3.20)$ respectively. Under the assumption that each branch point takes the form $\left(0, r_{j}\right)$ for $1 \leq j<t$ and with the same notations as in (4.1) and (4.2), the exponents $\delta_{t}:=\alpha_{1}\left(2 \widetilde{n}_{1}+p_{1}\right)$ and $\delta_{t}^{\prime}:=\frac{m_{t}^{\prime}}{m_{t}} \delta_{t}$ for $t>1$. When $t=1$, the exponents in (5.6) becomes $\delta_{1}:=2 \widetilde{n}_{1}+p_{1}$ and $\delta_{1}^{\prime}:=2 \widetilde{n}_{1}^{\prime}+p_{1}^{\prime}$. The reason is simply that $\exists d \in \mathbf{N}$ such that $|x y|\left|X_{1}\right|^{p_{1}}\left|Y_{1}\right|^{p_{1}^{\prime}} \sim\left|X_{t}\right|^{\delta_{t}}\left|Y_{t}\right|^{\delta_{t}^{\prime}}$ on 
$\operatorname{supp} \varphi_{0}^{t}$ whereas $\left|x y \| X_{1}\right|^{p_{1}}\left|Y_{1}\right|^{p_{1}^{\prime}} \sim\left|X_{t}\right|^{\delta_{t}}$ or $\left|Y_{t}\right|^{\delta_{t}^{\prime}}$ in the case of the terminal branch point $\left(0, r_{t}\right)$ or $\left(s_{t}, 0\right)$ on $\operatorname{supp} \varphi_{r}^{t}$ with $t \geq 1$.

In order to control the phases $\lambda P(x, y)$ of the operators $T_{0}^{t}$ and $T_{r}^{t}$ with $t \geq 1$, we make a scale transform $\left(\mathcal{X}_{t}, \mathcal{Y}_{t}\right)=\mathcal{L}\left(X_{t}, Y_{t}\right):=\left(\lambda^{\sigma_{1}} X_{t}, \lambda^{\sigma_{2}} Y_{t}\right)$ for the operator $T_{0}^{t}$, or $\left(\mathcal{X}_{t}, \widetilde{\mathcal{Y}}_{t}\right):=\left(\lambda^{\sigma_{1}} X_{t}, \lambda^{\sigma_{2}} \widetilde{Y}_{t}\right)$ or $\left(\widetilde{\mathcal{X}}_{t}, \mathcal{Y}_{t}\right):=\left(\lambda^{\sigma_{1}} \widetilde{X}_{t}, \lambda^{\sigma_{2}} Y_{t}\right)$ for the operator $T_{r}^{t}$ in the case of the terminal branch point $\left(0, r_{t}\right)$ or $\left(s_{t}, 0\right)$ respectively. Here the exponents $\sigma_{1}$ and $\sigma_{2}$ of $\lambda$ are to be determined. In this way the estimates in (5.6) become:

$$
\lambda|P(x, y)| \leq C\left|\mathcal{X}_{t}\right|^{\delta_{t}}\left|\mathcal{Y}_{t}\right|^{\delta_{t}^{\prime}}, \quad \text { and } \quad \lambda|P(x, y)| \leq C\left|\mathcal{X}_{t}\right|^{\delta_{t}} \text { or } C\left|\mathcal{Y}_{t}\right|^{\delta_{t}^{\prime}}
$$

under the following restrictive equations on $\sigma_{1}$ and $\sigma_{2}$ :

$$
\delta_{t} \sigma_{1}+\delta_{t}^{\prime} \sigma_{2}=1, \quad \text { and } \quad \delta_{t} \sigma_{1}=1 \text { or } \delta_{t}^{\prime} \sigma_{2}=1
$$

for the operator $T_{0}^{t}$, and for the operator $T_{r}^{t}$ in the case of the terminal branch point $\left(0, r_{t}\right)$ or $\left(s_{t}, 0\right)$ respectively.

Let $(\bar{x}, \bar{y}):=\mathcal{T}_{1} \circ \cdots \circ \mathcal{T}_{t}\left(\left(\mathcal{X}_{t}, \mathcal{Y}_{t}\right)\right)$ with $\mathcal{T}_{j}$ being the sequence of monomial transforms as in (3.10) for $1 \leq j \leq t$. Here for the operator $T_{r}^{t}$, similar to the definition of the variable $\widetilde{Y}_{t}$ or $\widetilde{X}_{t}$ in (3.19) or (3.20) in the case of the terminal branch point $\left(0, r_{t}\right)$ or $\left(s_{t}, 0\right)$, the variable $\mathcal{Y}_{t}$ or $\mathcal{X}_{t}$ is defined as $\mathcal{Y}_{t}:=\widetilde{\mathcal{Y}}_{t}+r\left(\mathcal{X}_{t}\right)$ or $\mathcal{X}_{t}:=\widetilde{\mathcal{X}}_{t}+s\left(\mathcal{Y}_{t}\right)$ respectively. Let us consider a rectangle $R_{h}:=[h, 2 h] \times$ $[h, 2 h]$ on the $\left(\mathcal{X}_{t}, \mathcal{Y}_{t}\right)$-plane, $\left(\mathcal{X}_{t}, \widetilde{\mathcal{Y}}_{t}\right)$-plane or $\left(\widetilde{\mathcal{X}}_{t}, \mathcal{Y}_{t}\right)$-plane with $h>0$ whose image on the $(\bar{x}, \bar{y})$-plane is denoted as $\mathcal{R}_{h}:=\mathcal{T}_{1} \circ \cdots \circ \mathcal{T}_{t}\left(R_{h}\right)$. We choose $\bar{a}, \bar{b}, \overline{a^{\prime}}, \overline{b^{\prime}} \in \mathbf{R}$ such that the rectangle $\bar{I}:=[\bar{a}, \bar{b}] \times\left[\overline{a^{\prime}}, \overline{b^{\prime}}\right] \subset \mathcal{R}_{h}$. Suppose the rectangle $\bar{I}$ corresponds to a rectangle $I:=[a, b] \times\left[a^{\prime}, b^{\prime}\right]$ on the $(x, y)$-plane under the scale transform $\mathcal{L}$, i.e., $\bar{I}=\mathcal{L}(I)$. Let $\delta_{1}$ and $\delta_{2}$ denote the horizontal and vertical magnitudes of $\mathcal{R}_{h}$ respectively. Then similar to the derivation of (5.2), $\exists h>0$ such that we can prove the estimates

$$
(\bar{b}-\bar{a})\left(\overline{b^{\prime}}-\overline{a^{\prime}}\right) \leq \delta_{1} \delta_{2} \leq C|\overline{x y}| \bar{\Gamma}_{t}^{2} \sim\left|\mathcal{X}_{t}\right|^{2 \mu_{t}}\left|\mathcal{Y}_{t}\right|^{2 \mu_{t}^{\prime}} \leq(2 h)^{2\left(\mu_{t}+\mu_{t}^{\prime}\right)}
$$

for the operator $T_{0}^{t}$ with $\bar{\Gamma}_{t}:=\prod_{j=1}^{t-1}\left(\mathcal{Y}_{j}-r_{j}\right)$ for $t>1$ and $\Gamma_{1}:=1$. For the operator $T_{r}^{t}$ in the case of the terminal branch point $\left(0, r_{t}\right)$ or $\left(s_{t}, 0\right)$, the estimate (5.8) takes the form $(\bar{b}-\bar{a})\left(\overline{b^{\prime}}-\overline{a^{\prime}}\right) \leq C|\overline{x y}| \widetilde{\mathcal{Y}}_{t}^{2} \bar{\Gamma}_{t}^{2} \sim\left|\mathcal{X}_{t}\right|^{2 \mu_{t}}\left|\widetilde{\mathcal{Y}}_{t}\right|^{2} \leq$ $(2 h)^{2\left(\mu_{t}+1\right)}$ or $(\bar{b}-\bar{a})\left(\overline{b^{\prime}}-\overline{a^{\prime}}\right) \leq C|\overline{x y}| \widetilde{\mathcal{X}}_{t}^{2} \bar{\Gamma}_{t}^{2} \sim\left|\widetilde{\mathcal{X}}_{t}\right|^{2}\left|\mathcal{Y}_{t}\right|^{2 \mu_{t}^{\prime}} \leq(2 h)^{2\left(\mu_{t}^{\prime}+1\right)}$ respectively. This indicates an estimate on the area of the rectangle $I$ as $(b-a)\left(b^{\prime}-a^{\prime}\right) \leq\left|X_{t}\right|^{2 \mu_{t}}\left|Y_{t}\right|^{2 \mu_{t}^{\prime}} \leq(2 h)^{2\left(\mu_{t}+\mu_{t}^{\prime}\right)} \lambda^{-2\left(\mu_{t} \sigma_{1}+\mu_{t}^{\prime} \sigma_{2}\right)}$ for the operator $T_{0}^{t}$, and $(b-a)\left(b^{\prime}-a^{\prime}\right) \leq\left|X_{t}\right|^{2 \mu_{t}}\left|\widetilde{Y}_{t}\right|^{2} \leq(2 h)^{2\left(\mu_{t}+1\right)} \lambda^{-2\left(\mu_{t} \sigma_{1}+\sigma_{2}\right)}$ or $(b-$ $a)\left(b^{\prime}-a^{\prime}\right) \leq\left|\widetilde{X}_{t}\right|^{2}\left|Y_{t}\right|^{2 \mu_{t}^{\prime}} \leq(2 h)^{2\left(\mu_{t}^{\prime}+1\right)} \lambda^{-2\left(\sigma_{1}+\mu_{t}^{\prime} \sigma_{2}\right)}$ for the operator $T_{r}^{t}$ in the case of the terminal branch point $\left(0, r_{t}\right)$ or $\left(s_{t}, 0\right)$ respectively. Thus if we define 
$f(x):=\chi_{[a, b]}(x)$ and $g(y):=\chi_{\left[a^{\prime}, b^{\prime}\right]}(y)$, then with the constant $C_{h}$ depending on $h$, the estimate

$$
\|f\|\|g\| \leq C_{h} \lambda^{-\left(\mu_{t} \sigma_{1}+\mu_{t}^{\prime} \sigma_{2}\right)}=C_{h} \lambda^{-1 /\left[2\left(\Delta_{t}+1\right)\right]}
$$

holds for the operator $T_{0}^{t}$, and the estimate

$$
\begin{aligned}
& \|f\|\|g\| \leq C_{h} \lambda^{-\left(\mu_{t} \sigma_{1}+\sigma_{2}\right)}=C_{h} \lambda^{-1 /\left[2\left(\Delta_{t}+1\right)\right]} \quad \text { or } \\
& \|f\|\|g\| \leq C_{h} \lambda^{-\left(\sigma_{1}+\mu_{t}^{\prime} \sigma_{2}\right)}=C_{h} \lambda^{-1 /\left[2\left(\Delta_{t}^{\prime}+1\right)\right]}
\end{aligned}
$$

holds for the operator $T_{r}^{t}$ in the case of the terminal branch point $\left(0, r_{t}\right)$ or $\left(s_{t}, 0\right)$ respectively under the following restrictive equations on $\sigma_{1}$ and $\sigma_{2}$ :

$$
\begin{gathered}
2\left(\mu_{t} \sigma_{1}+\mu_{t}^{\prime} \sigma_{2}\right)=1 /\left(\Delta_{t}+1\right), \quad \text { and } \\
2\left(\mu_{t} \sigma_{1}+\sigma_{2}\right)=1 /\left(\Delta_{t}+1\right) \quad \text { or } 2\left(\sigma_{1}+\mu_{t}^{\prime} \sigma_{2}\right)=1 /\left(\Delta_{t}^{\prime}+1\right)
\end{gathered}
$$

corresponding to the estimates in (5.9) and (5.10). Now the exponents $\sigma_{1}$ and $\sigma_{2}$ of $\lambda$ in the scale transform $\mathcal{L}$ can be determined from the restrictive equations (5.7) and (5.11).

Next we consider a rectangle $R_{c}:=\left[c_{1}, c_{2}\right] \times\left[c_{1}^{\prime}, c_{2}^{\prime}\right]$ on the $\left(\mathcal{X}_{t}, \mathcal{Y}_{t}\right)$-plane, $\left(\mathcal{X}_{t}, \widetilde{\mathcal{Y}}_{t}\right)$-plane or $\left(\widetilde{\mathcal{X}}_{t}, \mathcal{Y}_{t}\right)$-plane such that $\mathcal{T}_{1} \circ \cdots \circ \mathcal{T}_{t}\left(R_{c}\right) \subset \bar{I} \subset \mathcal{R}_{h}$. Here for the operator $T_{0}^{t}$, the $c_{2}^{\prime}>c_{1}^{\prime}>0$ satisfy $\exists \bar{x}_{0} \in[\bar{a}, \bar{b}]$ such that $c_{2}^{\prime}-c_{1}^{\prime} \leq$ $\left|\mathcal{Y}_{t}\left(\bar{x}_{0}, \overline{b^{\prime}}\right)-\mathcal{Y}_{t}\left(\bar{x}_{0}, \overline{a^{\prime}}\right)\right|$. Then $\exists h>0$ such that the following estimates hold on $\bar{I}$ as per Lemma 4.2 and Lemma 4.1:

$$
\left|\mathcal{Y}_{t}\left(\bar{x}_{0}, \overline{b^{\prime}}\right)-\mathcal{Y}_{t}\left(\bar{x}_{0}, \overline{a^{\prime}}\right)\right|=\int_{\overline{a^{\prime}}}^{\overline{b^{\prime}}}\left|\frac{\partial \mathcal{Y}_{t}}{\partial \bar{y}}\right| d \bar{y} \leq C \int_{\overline{a^{\prime}}}^{\overline{b^{\prime}}} \frac{\left|\mathcal{Y}_{t}\right|}{\left|\bar{y} \bar{\Gamma}_{t}\right|} d \bar{y} \leq \frac{2 C h\left(\overline{b^{\prime}}-\overline{a^{\prime}}\right)}{\inf _{R_{h}}\left|\bar{y} \bar{\Gamma}_{t}\right|} .
$$

The above two estimates yield a lower bound: $\overline{b^{\prime}}-\overline{a^{\prime}} \geq \frac{\left(c_{2}^{\prime}-c_{1}^{\prime}\right)}{2 C h} \inf _{R_{h}}\left|\bar{y} \bar{\Gamma}_{t}\right|$. Similarly we can adjust $c_{1}^{\prime}$ and $c_{2}^{\prime}$ such that $\bar{b}-\bar{a} \geq \frac{\left(c_{2}^{\prime}-c_{1}^{\prime}\right)}{2 C^{\prime} h} \inf _{R_{h}}\left|\bar{x} \bar{\Gamma}_{t}\right|$. For the operator $T_{r}^{t}$ in the case of the terminal branch point $\left(0, r_{t}\right)$ or $\left(s_{t}, 0\right)$, we substitute the variable $\widetilde{\mathcal{Y}}_{t}$ or $\widetilde{\mathcal{X}}_{t}$ for the variable $\mathcal{Y}_{t}$ respectively in the above estimates; and accordingly, we also have to substitute the $\bar{\Gamma}_{t}$ in the above estimates by $\widetilde{\mathcal{Y}}_{t} \bar{\Gamma}_{t}$ or $\widetilde{\mathcal{X}}_{t} \bar{\Gamma}_{t}$ respectively. Thus similar to the process of deriving the estimates (5.9) and (5.10), after proving the following lower bounds on the area of the rectangle $I$ as $(b-a)\left(b^{\prime}-a^{\prime}\right) \geq C_{h}^{\prime} \lambda^{-2\left(\mu_{t} \sigma_{1}+\mu_{t}^{\prime} \sigma_{2}\right)}$ for the operator $T_{0}^{t}$, and $(b-a)\left(b^{\prime}-a^{\prime}\right) \geq C_{h}^{\prime} \lambda^{-2\left(\mu_{t} \sigma_{1}+\sigma_{2}\right)}$ or $(b-a)\left(b^{\prime}-a^{\prime}\right) \geq C_{h}^{\prime} \lambda^{-2\left(\sigma_{1}+\mu_{t}^{\prime} \sigma_{2}\right)}$ for the operator $T_{r}^{t}$ in the case of the terminal branch point $\left(0, r_{t}\right)$ or $\left(s_{t}, 0\right)$ respectively, we can prove that $\exists h, C_{h}>0$ such that:

$$
\left|\left\langle T^{t} f, g\right\rangle\right| \geq C_{h} \lambda^{-1 /\left(\Delta_{t}+1\right)} \quad \text { or } \quad\left|\left\langle\widetilde{T}^{t} f, g\right\rangle\right| \geq C_{h} \lambda^{-1 /\left(\Delta_{t}^{\prime}+1\right)}
$$


under the same restrictive equations on $\sigma_{1}$ and $\sigma_{2}$ as in (5.11). Here the operator $T^{t}$ in (5.12) denotes the operator $T_{0}^{t}$ or the operator $T_{r}^{t}$ in the case of the terminal branch point $\left(0, r_{t}\right)$; whereas the operator $\widetilde{T}^{t}$ in (5.12) denotes the operator $T_{r}^{t}$ in the case of the terminal branch point $\left(s_{t}, 0\right)$. Summarizing the estimates in (5.9), (5.10) and (5.12), we have:

$$
\left|\left\langle T^{t} f, g\right\rangle\right| \geq C_{h} \lambda^{-1 /\left[2\left(\Delta_{t}+1\right)\right]}\|f\|\|g\| \text { or }\left|\left\langle\widetilde{T}^{t} f, g\right\rangle\right| \geq C_{h} \lambda^{-1 /\left[2\left(\Delta_{t}^{\prime}+1\right)\right]}\|f\|\|g\|
$$

with the operators $T^{t}$ and $\widetilde{T}^{t}$ defined as in (5.12). Thus we verified the sharpness of the estimates in (5.4) and (5.5). In particular when $t=1$, we need to substitute $\Delta_{\kappa}$ for $\Delta_{t}$ or $\Delta_{t}^{\prime}$ in the above estimates.

Lemma 4.4 further indicates that $\left\|T_{0}^{t}\right\|,\left\|T_{r}^{t}\right\| \leq C \lambda^{-1 /\left[2\left(\Delta_{\kappa}+1\right)\right]}$. Due to the finiteness of the algorithm of operator decompositions in Section 3.3, as well as the sharpness of the estimate on the operator $T_{0}$ in (3.27), the operator $T_{\kappa}$ in (3.27) satisfies the sharp estimate $\left\|T_{\kappa}\right\| \leq C \lambda^{-1 /\left[2\left(\Delta_{\kappa}+1\right)\right]}$.

Finally, as per the operator decomposition in (3.24) and the definition in (3.9), we can obtain the sharp estimate $\left\|T_{\lambda}\right\| \leq C \lambda^{-1 /[2(\Delta+1)]}$, which is the first conclusion of the theorem as in (1.3).

We would like to draw attentions to a further research direction, which is to generalize the theorem to high-dimensional oscillatory integral operators.

\section{Acknowledgements}

The author would like to express his gratitude to the referees for carefully reviewing the manuscript and making valuable suggestions.

\section{References}

[1] V. Arnold, S. Guseĭn-Zade and A. Varchenko, Singularities of differentiable maps, Vol. II, Monographs in mathematics 83, Birkhäuser, 1988.

[2] G. Beylkin, Imaging of discontinuities in the inverse scattering problem by inversion of a causal generalized Radon transform, J. Math. Phys. 26 (1985), no. 1, 99-108.

[3] L. Carleson and P. Sjölin, Oscillatory integrals and a multiplier problem for the disc, Studia Math. 44 (1972), 287-299. (errata insert).

[4] M. Cotlar, A combinatorial inequality and its applications to $L^{2}$-spaces, Rev. Mat. Cuyana 1 (1955), 41-55 (1956).

[5] L. Hörmander, Oscillatory integrals and multipliers on $F L^{p}$, Ark. Mat. 11 (1973), 1-11.

[6] G. Kempf, F. Knudsen, D. Mumford and B. Saint-Donat, Toroidal embeddings. I. Lecture Notes in Math., 339, Springer, Berlin, 1973

[7] S.-M. Ma, The Newton polygon and oscillatory integral operators, Ph.D. Thesis, Columbia University, 1995.

[8] $\frac{}{89-98}$ A survey on oscillatory integral operators, Anal. Theory Appl. 19 (2003), no. 1, 
[9] S.-M. Ma, A fast algorithm for curve singularities, Bull. Austral. Math. Soc. 69 (2004), no. 3, 403-414.

[10] Y. Meyer, Wavelets and operators, Different perspectives on wavelets (San Antonio, TX, 1993), 35-58, Proc. Sympos. Appl. Math., 47, Amer. Math. Soc., Providence, RI, 1993.

[11] D. Phong and E. Stein, The Newton polyhedron and oscillatory integral operators, Acta Math. 179 (1997), no. 1, 105-152.

[12] A. Varchenko, Newton polyhedra and estimation of oscillating integrals, Funct. Anal. Appl. 10 (1976), 175-196. 